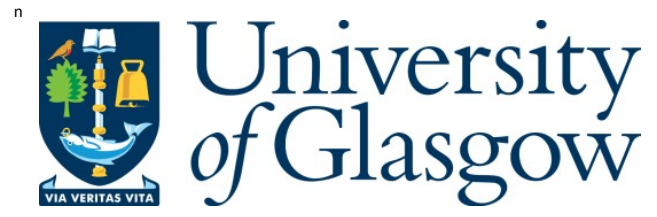

Dehaeze, F., Baverstock, K. D., and Barakos, G. N. (2015) CFD simulation of flapped rotors. A eronautical J ournal, 119(1222), pp. 1561-1583. 1561.

There may be differences between this version and the published version. Y ou are advised to consult the publisher's version if you wish to cite from it.

http://eprints.gla.ac.uk116482/

Deposited on: 04 M arch 2016

Enlighten - R esearch publications by members of the U niversity of Glasgow http://eprints.gla.ac.uk 


\title{
CFD SIMULATION OF FLAPPED ROTORS
}

\author{
F. Dehaeze $\mathrm{D}^{\dagger}$ 1, K.D. Baverstock ${ }^{\ddagger}$ and G.N. Barakos ${ }^{\dagger}$ \\ † CFD Laboratory, School of Engineering \\ University of Liverpool, L69 3GH, U.K. \\ http://www.liv.ac.uk/cfd/ \\ $\ddagger$ AgustaWestland Ltd \\ Yeovil BA20 2YB, UK \\ http://www.agustawestland.com
}

Email: Florent.Dehaeze@agustawestland.com,Karl.Baverstock@agustawestland.com,G.Barakos@liverpool.ac.uk

\begin{abstract}
The use of active trailing edge flaps on rotors may lead to performance benefits as well as noise and vibration reduction. In this work, the HMB CFD solver is used, and the trailing edge flaps are modelled using a combination of surface and mesh deformation. Starting from a baseline blade design, the flap is first assessed using dMdt computations, that account for the simultaneous variations of pitch and Mach around the azimuth. It was shown that enhanced lift was obtained while inspection of the moment coefficient showed negative damping for the flap for a limited set of conditions. Due to the 2D formulation, $\mathrm{dMdt}$ computations are fast to perform and can be used to inform codes predicting the rotor performance. The flap was then assessed in hover, and only allowed for limited improvement in blade performance at high thrust. In forward flight, the flap was actuated in a 1-per-rev fashion and was found to have a strong effect on the loads on the retreating side. The effect on the moments was even stronger. The flight envelope of the blade was explored, and clean and flapped cases were compared. The most noticeable changes occur at high and medium thrust. The CFD method was found to be efficient and robust, without any substantial penalties in CPU time over the tested conditions.
\end{abstract}

\section{NOMENCLATURE}

$c \quad$ Chord length

$C_{D} \quad$ Aerofoil drag coefficient

$C_{L} \quad$ Aerofoil lift coefficient

$C_{M} \quad$ Aerofoil pitching moment coefficient

$C_{P} \quad$ Pressure coefficient, $C_{P}=\frac{P-P_{\infty}}{\frac{1}{2} \rho_{\infty} V_{l o c}^{2}}$

$C_{Q} \quad$ Rotor torque coefficient, $C_{Q}=\frac{Q}{\frac{1}{2} \rho_{\infty} \omega^{2} \pi R^{5}}$

$C_{T} \quad$ Rotor thrust coefficient, $C_{T}=\frac{T}{\frac{1}{2} \rho_{\infty} \omega^{2} \pi R^{4}}$

$\vec{F}_{i}, \vec{F}_{v} \quad$ Inviscid and viscous fluxes

$k \quad$ Reduced frequency, $k=\frac{\omega c}{2 V_{\infty}}$

$k-\omega$ SST Menter's $k-\omega$ Shear-Stress Transport [9]

$L_{q} \quad$ Sectional normal loading

$L_{q} \quad$ Sectional pitching moment loading (around the blade axis)

$L_{q} \quad$ Sectional torque loading

$a_{\infty} \quad$ Free stream speed of sound

$M_{\infty} \quad$ Free stream Mach number

$M^{2} C_{m}$ Mach-scaled sectional pitching moment coefficient (around the blade axis), $M 2 C_{m}=\frac{L_{m}}{\frac{1}{2} \rho_{\infty} a_{\infty}^{2} c^{2}}$

$M^{2} C_{n}$ Mach-scaled sectional normal force coefficient, $M 2 C_{n}=\frac{L_{n}}{\frac{1}{2} \rho_{\infty} a_{\infty}^{2} c^{2}}$
$M^{2} C_{q}$ Mach-scaled sectional torque coefficient, $M 2 C_{q}=$ $\frac{L_{q}}{\frac{1}{2} \rho_{\infty} a_{\infty}^{2} c^{2}}$

$M_{H} \quad$ Flap hinge moment

$P \quad$ Local pressure

$P_{\infty} \quad$ free stream pressure pressure

$R \quad$ Rotor radius

$r \quad$ Current spanwise location

$R e_{\infty} \quad$ Reynolds number based on the free stream velocity: $R e_{\infty}=\frac{\rho c V_{\infty}}{\mu_{\infty}}$

$\mathbf{R}_{i, j, k} \quad$ Flux residuals at cell $(i, j, k)$

$\vec{S} \quad$ Source term

Q Rotor torque

$T \quad$ Rotor thrust

$\vec{u}_{h} \quad$ Local velocity field in the rotor-fixed frame of reference

$u, v, w \quad$ Velocity components

$<V>$ Average velocity

$V(t) \quad$ Time dependent control volume

$V_{\infty} \quad$ Local velocity

$V_{0} \quad$ Average component of the local velocity

$V_{1 s} \quad$ Sine component of the local velocity

$V_{l o c} \quad$ Free stream velocity

\footnotetext{
${ }^{1}$ F. Dehaeze is now employed by AgustaWestland Ltd
} 
$\mathbf{w}_{i, j, k} \quad$ Discretise conserved variables vector

$\overrightarrow{\mathbf{w}} \quad$ Conserved variables vector

$\alpha \quad$ Incidence

$\alpha_{i} \quad$ Modal amplitude coefficient for the i-th eigenmode

$\bar{\alpha} \quad$ Peak-to-peak variation of the incidence

$\overline{\delta_{f}} \quad$ Peak-to-peak variation of the flap deployment

$\bar{x} \quad$ Peak-to-peak variation of the aerofoil location in the translation motion

$\Delta M^{2} C_{m}^{*}$ Variation of the sectional pitching moment coefficient, defined in Equation (9)

$\Delta M^{2} C_{n}^{*}$ Variation of the sectional normal force coefficient, defined in Equation (7)

$\Delta M^{2} C_{q}^{*}$ Variation of the sectional torque coefficient, defined in Equation (8)

$\Delta Z \quad$ Flapping deflection

$\delta_{f} \quad$ Flap deployment angle

$\mu \quad$ Forward flight advance ratio

$\mu_{\infty} \quad$ Air viscosity at free stream conditions

$\omega \quad$ Rotor rotational speed

$\phi \quad$ Blade deformed shape

$\phi_{0} \quad$ Blade shape under rotational loading

$\phi_{i} \quad$ Mass-scaled modal deformation of the i-th eigenmode

$\psi \quad$ Azimuth

$\rho \quad$ Air density

$\rho_{\infty} \quad$ Free stream air density

$\sigma \quad$ Rotor solidity

$\theta_{\text {elas }} \quad$ Elastic torsion

$\xi_{\text {flap }} \quad$ Flap damping coefficient, defined in Equation (5)

$\xi_{\text {rot }}$ Rotational damping coefficient, defined in Equation (4)

$\xi_{\text {trans }}$ Translational damping coefficient, defined in Equation (6)

BVI Blade-Vortex Interaction

CFD Computional Fluid Dynamics

CPU Central Processing Unit

MUSCL Monotonic Upstream-Centered Scheme for Conservation Laws

\section{INTRODUCTION}

Active devices are seen as a way to improve the blade performance by producing more optimised conditions for both the transonic speeds on the advancing side and the low speed at high angles of attack on the retreating side. They are also seen as a means to reduce the vibration level and the emitted noise. During the last few years, many techniques have been tested, such as Gurney flaps [2,-4, 11], trailing-edge flaps [5, 15, 18], higher harmonic control [14,19], and active twist [7].

Many works targeted one flight condition, mainly a slow BVI descent flight [4, 7, 11]. Other works also focused on one single high-speed forward flight condition [2, 5, 15]. As a result, benefits could be achieved in those particular conditions, but no work so far showed the effect of the flaps over the envelope using a modern helicopter rotor.

Very few studies on active flaps aimed to exploring the flight domain. Wind tunnel tests were performed by Straub et al. [18] on a SMART blade to assess the effect of adding a trailing edge flap. That work highlighted the benefits of using flaps to lower the rotor vibration or noise on the whole flight domain, but showed little improvement in performance. Flight tests conducted by Airbus Helicopters [12] highlighted a reduction of the 4/Rev vibration levels over the whole speed range in forward flight, using the BK-117 helicopter equipped with trailing edge flaps. This reduction reached $80 \%$ at a speed of $95 \mathrm{KN}$. A study by Ravichandran et al. [13] was carried on the whole flight domain of a UH-60A rotor using a trailing-edge flap. A first study on the hovering rotor revealed that deploying the flaps would increase the rotor figure of Merit by deforming the blade, and this effect was increased when the blade was made softer in torsion. The study in forward flight at high advance ratios revealed that using a $1 / \operatorname{Rev}$ and $2 / \operatorname{Rev}$ actuation sequence could improve the performance by reducing the power requirements by up to $5 \%$. One high-speed condition was also chosen to test the effectiveness of flaps at reducing the levels of hub load vibrations, using a combination of 3 to 5/Rev actuation. A reduction of $80 \%$ in the vibratory loads was achieved. However, such high reduction in terms of vibration led to increased power requirements. It was found that for a particular actuation at 1-5/Rev, benefits in both performance and vibration levels. Similar work has been performed using Gurney flaps [3] or tabs [8].

This work proposes to study the effect of trailing edge flaps in the whole flight domain for a modern mid-sized helicopter main rotor using CFD. After the CFD solver is presented, selected sections of the rotor are studied in dynamic stall conditions, and using dMdt simulations, presented in Section 3.1.2 It is followed by simulations of the whole rotor in hover and forward flight. 


\section{CFD METHOD}

The Helicopter Multi-Block (HMB) code, developed at Liverpool, is used as the CFD solver for the present work. It solves the Navier-Stokes equations in integral form using the arbitrary Lagrangian Eulerian (ALE) formulation for timedependent domains with moving boundaries:

$$
\frac{d}{d t} \int_{V(t)} \overrightarrow{\mathbf{w}} d V+\int_{\partial V(t)}\left(\vec{F}_{i}(\overrightarrow{\mathbf{w}})-\vec{F}_{v}(\overrightarrow{\mathbf{w}})\right) \vec{n} d S=\vec{S}
$$

where $V(t)$ is the time dependent control volume, $\partial V(t)$ its boundary, $\overrightarrow{\mathbf{w}}$ is the vector of conserved variables $[\rho, \rho u, \rho v, \rho w, \rho E]^{T} . \vec{F}_{i}$ and $\vec{F}_{v}$ are the inviscid and viscous fluxes, including the effects of the time dependent domain. For forward flying rotor simulations, a moving grid approach is used and the source term is set to $\vec{S}=[0, \overrightarrow{0}, 0]^{T}$.

The Navier-Stokes equation are discretised using a cellcentred finite volume approach on a multi-block grid, leading to the following equations:

$$
\frac{\partial}{\partial t}\left(\mathbf{w}_{i, j, k} V_{i, j, k}\right)=-\mathbf{R}_{i, j, k}\left(\mathbf{w}_{i, j, k}\right)
$$

where $\mathbf{w}$ represents the cell variables and $\mathbf{R}$ the residuals. $i, j$ and $k$ are the cell indices and $V_{i, j, k}$ is the cell volume. Osher's [10] upwind scheme is used to discretise the convective terms and MUSCL variable interpolation is used to provide third order accuracy, and the Van Albada limiter [20] is used to reduce the oscillations near steep gradients. Menter's $k-\omega$ SST turbulence model [9] was used for all simulations.

Temporal integration is performed using an implicit dualtime step method. The linearised system is solved using the generalised conjugate gradient method with a block incomplete lower-upper (BILU) pre-conditioner [1].

Multi-block structured meshes are used for HMB. These meshes are generated using ICEM-Hexa ${ }^{\mathrm{TM}}$ of Ansys. The multi-block topology allows for an easy sharing of the calculation load for parallel computing. For rotor flows, a typical multi-block topology used in the University of Liverpool is described in [16]. A C-mesh is used around the blade and this is included in a larger $\mathrm{H}$ structure which fills up the rest of the computational domain. The block boundaries on a forward flying ONERA 7A rotor is shown in black in Figure 1

The mesh deformation method account for the flaps is described by Steijl et al. [17]. In forward flight, the blade deformation was prescribed. The deformation were based on in-flight measurements, and were described based on a modal analysis of the blade. The blade shape was therefore seen as:

$$
\phi=\phi_{0}+\sum_{i=1}^{n_{m}} \alpha_{i} \phi_{i}
$$

where $\phi$ is the blade shape, $\phi_{0}$ the shape of the blade undeformed and $\phi_{i}$ is the i-th eigenmode of the blade. The amplitude coefficients $\alpha_{i}$ were obtained from in-flight measurements.

The application of the blade deformation requires the CFD mesh to deform. A method developed for HMB first deforms the blade surface using the Constant Volume Tetrahedron method, then obtains the updated block vertex positions via spring analogy and finally generates the full mesh via Transfinite Interpolation. It is extensively described in [6]. The Transfinite Interpolation first interpolates the block edges and faces from the new vertex position and then interpolates the full mesh from the surfaces. This method uses the properties of multi-block meshes and maintains its efficiency as the number of blocks increases, particularly in the spanwise blade direction.

\section{Results And Discussion}

A main 5-bladed rotor, representative of a modern main rotors was used in this work. The blade planform can be seen in Figure 2, and had a nominal twist of 11 degrees $/ R$. Three flaps were added to the blade, located near the antinodes of the second and third flapping modes. The blade uses three modern rotorcraft aerofoil section along the span.

\subsection{D Aerofoil study}

\subsubsection{Pitching aerofoils}

A first test was performed on pitching aerofoils. Two sections of the main rotor blade were tested: one centered at $r / R=0.6$ and another one at $r / R=0.8$. All test cases were run at $M_{\infty}=0.3$ and $R e_{\infty}=4.2 \times 10^{6}$. Eight conditions were chosen for the pitching simulations and are shown in Table 1. At all conditions, three flap positions were tested: $\delta_{f}=0$ degree, $\delta_{f}=-2.5$ degrees and $\delta_{f}=4.5$ degrees. A moving flap was also tested on the inboard section, using an actuation of $\delta_{f}=3-3 \sin (\psi)$ degrees.

The aerodynamic damping coefficient of the pitching motion during a cycle, defined as:

$$
\xi_{\text {rot }}=-\frac{1}{\frac{1}{2} \rho_{\infty}<V>^{2} c^{3} \pi \bar{\alpha}^{2}} \oint_{\text {cycle }} M_{\text {pitch }} d \alpha
$$

was extracted and is shown in Figure 3 a for the inboard section and $3 \mathrm{~b}$ for the outboard section. Only one case led to a negative damping coefficient, corresponding to the inboard section with $\alpha=14-5 \sin (\psi)$ degrees, and the flap position of $\delta_{f}=-2.5$ degrees. All other cases led to positive damping coefficients, highlighting the stability of the aerofoil in pitching motions.

For the cases where the flap was actuated, the damping coefficient of the flap rotation around his hinge, defined as:

$$
\xi_{\text {flap }}=-\frac{1}{\frac{1}{2} \rho_{\infty}<V>^{2} c^{3} \pi \bar{\alpha}^{2}} \oint_{\text {cycle }} M_{H} d \delta_{f}
$$

was extracted and is shown in Figure $3 \mathrm{c}$. Negative values highlight flap instabilities. For the second case where $\alpha=10-10 \sin (\psi)$ degrees the negative damping coefficient was close to 0 and could easily be compensated by the structural and mechanical losses, in the fourth case where $\alpha=14-14 \sin (\psi)$ degrees, the damping was clearly negative.

\subsection{2 dMdt Simulations}

dMdt simulations consider a translating and pitching aerofoil, aiming at getting closer to the conditions undergone by a rotor section without the cost of a full $3 \mathrm{D}$ rotor simulation. 
Such simulations can be used to assess the aerodynamic models employed in blade element models, which usually obtain the sectional lift and drag based on aerofoil polars, corrected for the pitching motion. In dMdt simulations, changes in incidence are obtained by modifying the pitch angle, while changes in velocity are obtained by modifying the translational speed, as shown in Figure 4. Unlike blade element models, which usually only account for the incidence, Mach number, Reynolds number and have a model to assess the influence of the variation of the incidence, dMdt simulations also take into account the variation of the Mach and Reynolds number.

Eight simulations were carried out, whose conditions are shown in Table 2 These conditions are representative of sections at $r / R=0.6$ (Cases 1-4) and at $r / R=0.8$ (Cases 5-8). Figure 5 shows the flow field evolution with the azimuth: the contours show the pressure coefficient. The wake velocity in the aerofoil frame of reference was also extracted at two vertical lines behind the aerofoil. The pressure coefficients $C_{P}$ were extracted using the local velocity: $C_{P}=\frac{P-P_{\infty}}{\frac{1}{2} \rho_{\infty} V_{l o c}^{2}}$, with $V_{l o c}(\psi)=V_{0}+V_{1 s} \sin (\psi)$. A shock can clearly be seen on the advancing side at $\psi=90$ degrees, while high suction appears on the retreating side at $\psi=270$ degrees.

The lift, drag and moment coefficients were extracted, and are shown in Figure 6 for Cases 1-4, and in Figure 7 for Cases 5-8. The most noticeable feature is the negative drag coefficient on the retreating side when the flap is deployed. This comes from the wake being trailed from the trailing edge at higher speeds reaching the aerofoil when it slows down. As the moment coefficient shows, there is no stall on the retreating side.

Symbols were also added in the lift, drag and moment coefficients plots, showing the force and moment predictions if a steady flow is adopted, by extracting the lift, drag and moment coefficients on static polar at similar conditions, when available. In Cases 1-4, while the match was fair on the advancing side, the predictions in the conditions of the retreating side proved off. In Cases 5-8, the coefficients from the dMdt simulations and the static ones did not match. This highlights the importance in taking into account the dynamic effects due to the changes in pitch and speed in blade element models.

Again, the aerodynamic damping coefficients in translation and pitching were computed. The aerodynamic damping coefficient in pitching was defined in Equation (4), and the aerodynamic damping coefficients in translation is defined as follows:

$$
\xi_{\text {trans }}=\frac{1}{\frac{1}{2} \rho_{\infty}<V>^{2} c^{2} \pi \bar{x}^{2}} \oint_{\text {cycle }} F_{\text {drag }} d x
$$

They are shown for each case in Figure 8 The translational variations always proved stable, with a high damping coefficient in all cases. On the aerodynamic pitching damping side, all cases also showed a positive damping, but when the cycle was simulated with a fully deployed flap (Cases 3 and 7), the damping coefficient was lower, becoming small in Case 7. This goes against what was observed for pitching aerofoils where deploying the flap increased the damping coefficient.

\subsection{Main Rotor in Hover}

The main rotor, previously described, was equipped with flaps, as shown in Figure 2. The flaps were located around the anti-nodal locations of the second and third flapping modes, to allow for vibration control. In hover, all flaps were synchronised and were tested at two deployed positions: $\delta_{f}=$ -2.5 degrees and $\delta_{f}=4.5$ degrees. Three collective were tested. The evolution of the figure of Merit with the thrust coefficient is shown in Figure 10. Only 3 points were simulated for each flap setting, and trends were interpolated, as shown with the lines on the graph. Deploying the flaps up did not bring any improvement; however, setting the flaps at $\delta_{f}=4.5$ degrees improved the figure of Merit on a limited range of high thrust coefficients.

The distributions of the sectional thrust and torque were extracted for the medium collective case, and are shown in Figure 9 The sectional thrust and torque were nondimensionalised using their value at $r / R=0.75$ in the case without flaps. The location of the flaps is shown using dashdotted lines.The effect of the inboard flaps on the loading proved to be limited to the flap area and its close surroundings. On the other hand, the tip flap also modified the blade loading outboard of the flap all the way to the tip. This alteration of the loading will have an influence on the induced power, which might not be beneficial and could explain the limited benefit in performance when deploying the flaps.

\subsection{Main Rotor in Forward Flight}

The same rotor was simulated in forward flight to assess the effect of the flaps over the whole flight envelope. In total, six flights were simulated, at low and high speed at three different thrusts. A summary of the flight conditions is shown in Figures 11. For these simulations, the blade was considered rigid, and the rotor was run without flap actuation, or with all flaps actuated at $\delta_{f}=3-3 \sin (\psi)$ degrees. The main goal of the flap actuation was to alleviate the stall on the retreating side and improve the rotor performance.

For each flight, the sectional thrust, torque and pitching moments were extracted. Their values in the cases without flap were substracted from the values with flaps, and the result was divided by the maximum absolute value without flap:

$$
\begin{aligned}
\Delta M^{2} C_{n}^{*} & =\frac{M^{2} C_{n, \text { with flap }}-M^{2} C_{n, \text { without flap }}}{\max \left(\left|M^{2} C_{n, \text { with flap }}\right|\right)} \\
\Delta M^{2} C_{q}^{*} & =\frac{M^{2} C_{q, \text { with flap }}-M^{2} C_{q, \text { without flap }}}{\max \left(\left|M^{2} C_{q, \text { with flap }}\right|\right)} \\
\Delta M^{2} C_{m}^{*} & =\frac{M^{2} C_{m, \text { with flap }}-M^{2} C_{m, \text { without flap }}}{\max \left(\left|M^{2} C_{m, \text { with flap }}\right|\right)}
\end{aligned}
$$

The Mach-scaled sectional forces and moment, as well as resulting variation ratios, are shown for each flight in Figures 12,17

In most flights, a strong effect on the pitching moment was seen at the location of the flaps, with the pitching moment amplitudes increasing by $12 \%$ or more. At high thrust, the flaps delayed the effect of the stall on the retreating side. This can be seen in Figures 12 and 13, where the stall on the retreating side is clearly visible due to the drop in moments in Figures 12e and 13e. It can be seen that the stall is delayed in Figures 12f and 13f] where the red patches in the stall location indicate that the pitching moment did not drop 
as early. At medium thrust, the main effect of the flaps at low speed was to modify the blade-vortex interaction in the second quarter of the disk as seen in Figure14 where high variations of the $M^{2} C_{n}$ and $M^{2} C_{m}$ coefficients can be spotted, but at high speed, the flap mainly affected the thrust on the advancing side, and the stall on the retreating side as seen in Figure 15. At low thrust, the main effect of the flaps was to locally increase the thrust and the torque at the location of the flaps, as shown in Figures 16 17. It also had an effect on the blade-vortex interactions at the front of the disk. It was also seen that, in all conditions, the use of flaps on the blade could largely increase the levels of sectional pitching moment where the flaps are located, which would result in higher control loads.

A comparison of the resulting thrust and torque for the cases with and without flaps is shown in Figure 18. On this graph, each flight is shown with a symbol located at its thrust coefficient and advance-ratio. The colour of the symbol indicates, in percent, the variation of the thrust coefficient in Figure 18a, the variation of the torque coefficient in Figure $18 \mathrm{~b}$ and the difference between these two figures in Figure 18c While the obtained rotor thrust in the cases with and without flaps were not always matching due to the applied trim state, it can be seen that in some cases, some benefit can be obtained from the flaps. At high thrust and low speed, for a similar thrust, the flaps allowed for a reduction of $5 \%$ of the torque. At high thrust and high speed though, the thrust was kept similar but the required torque was largely increased. At medium thrust and low speed, the thrust was increased by $2.5 \%$ by actuating the flaps, while the torque was slightly reduced. At low thrust, no noticeable gain was achieved.

Using the conditions from Flight 2-21, prescribed blade deformations were also applied to the rotor, based on in-flight measurements. The applied deformations are shown in Figure 19. The same deformation had to be applied to the blades with and without flaps actuated, due to a lack of data for the flight with flaps. The new difference in sectional coefficient between the cases with and without actuated flaps is shown in Figure 20. The sectional loads for the case without flaps are also shown, using the same scale as the one used in Figure 15 The most noticeable change is the much lower sectional lift on the advancing side seen in Figure20a It can be seen that the actual difference in aerodynamic loads is very similar to the rigid case shown in Figure 15 meaning that the effect of the blade deformation on the sectional thrust and torque in both cases was similar. This, however, needs further investigation.

\section{Summary AND CONCLUSIONS}

A comprehensive analysis of flapped rotors has been carried out, showing various ways to assess the effect of flaps on a helicopter main rotor. On a selected main rotor designed for a middle-sized helicopter, analyses ranging from $2 \mathrm{D}$ pitching aerofoils to full simulations of a forward flying rotor were carried out.

The 2D analysis using the $\mathrm{dMdt}$ approach highlighted the need to take into account the dynamic effects when modelling the rotor using a simple blade element theory. They also proved quick and allowed to have a first estimate of the flap stability, without using expensive CFD simulations of the full rotor in forward flight.

A study of the full main rotor using CFD was then carried out. At first, the rotor was studied in hover, and it was highlighted that deploying the flaps at high thrust could allow for a small increase of the figure of Merit. This improvement, however, proved limited. Further analyses could however focus on only deploying part of the flaps, or at differentiated levels of deployment.

The focus was then moved to forward flying rotors, and the whole flight domain of a helicopter was studied. Despite trimming issues, it was highlighted that the flaps proved beneficial at high thrust and high lift, except when the blade on the retreating side is already deeply stalled and the flaps can not help recovering the flow field. The influence of the flaps on the rotor performance at lower thrust proved however limited.

\section{ACKNOWLEDGEMENTS}

The financial support via the RTVP project of AgustaWestland and the Business Innovation and Skills Department of UK is gratefully acknowledged.

\section{COPYRIGHT STATEMENT}

The authors confirm that they, and/or their company or organisation, hold copyright on all of the original material included in this paper. The authors also confirm that they have obtained permission, from the copyright holder of any third party material included in this paper, to publish it as part of their paper. The authors confirm that they give permission, or have obtained permission from the copyright holder of this paper, for the publication and distribution of this paper as part of the ERF2014 proceedings or as individual offprints from the proceedings and for inclusion in a freely accessible web-based repository.

\section{REFERENCES}

[1] O. Axelsson. Iterative Solution Methods. Cambridge University Press: Cambridge, MA, 1994.

[2] E.S. Bae and F. Gandhi. Upstream Active Gurney Flap for Rotorcraft Vibration Reduction. In American Helicopter Society 68th Annual Forum, Fort Worth, TX, May $1-32012$.

[3] E.S. Bae, F. Gandhi, and M. Maughmer. Optimally Scheduled Deployments of Miniature Trailing-Edge Effectors for Rotorcraft Power Reduction. In American Helicopter Society 65th Annual Forum, Grapevine, TX, May 27-29 2009.

[4] O. Bauchau, B.-Y. Min, and L. Sankar. A CFD-CSD Coupled-Analysis of HART-II Rotor Vibration Reduction Using Gurney Flaps. In American Helicopter Society 68th Annual Forum, Phoenix, AZ, May 11-13 2010.

[5] T.S. Birchette, F.K. Straub, E.W. Brouwers, R.C. Bussom, and L.R. Centolanza. Active Flap Design for an Advanced CH-47. In American Helicopter Society 69th Annual Forum, Phoenix, AZ, May 21-23 2014. 
[6] F. Dehaeze and G.N. Barakos. Mesh Deformation Method for Rotor Flows. Journal of Aircraft, 49(1):8292, January-February 2012. Accepted for publication.

[7] D.E. Fogarty, M.L Wilbur, M.K. Sekula, and D.D. Boyd. CFD/CSD Investigation of BVI Noise Reduction Using Higher Harmonic Active-Twist Control. In 38th European Rotorcraft Forum, Amsterdam, Netherlands, Paper No75, September 4-6 2012. .

[8] O. Léon, E. Hayden, and F. Gandhi. Rotorcraft Operating Envelope Expansion Using Extendable Chord Sections. In American Helicopter Society 65th Annual Forum, Grapevine, TX, May 27-29 2009.

[9] F.R. Menter. Two-Equation Eddy-Viscosity Turbulence Models for Engineering Applications. AIAA Journal, 32(8):1598-1605, 1994.

[10] S. Osher and S. Chakravarthy. Upwind Schemes and Boundary Conditions with Applications to Euler Equations in General Geometries. Journal of Computational Physics, 50:447-481, January-February 1983.

[11] A. Padthe. Simulataneous BVI Noise and Vibration Reduction in Rotorcraft Using Microflaps Including the Effect of Actuator Saturation. In American Helicopter Society 68th Annual Forum, Fort Worth, TX, May 1-3 2012.

[12] A. Rabourdin, J.-B. Maurice, O. Dietrich, and P. Konstanzer. Blue Pulse Active Rotor Control at Airbus Helicopters - New EC-145 Demonstrator \& Flight Test Results. In American Helicopter Society 70th Annual Forum, Montréal, Québec, May 20-22 2013.

[13] K. Ravichandran, I. Chopra, B.E. Wake, and B. Hein. Trailing-Edge Flaps for Rotor Performance Enhancement and Vibration Control. Journal of the American Helicopter Society, 58(2):1-13, April 2013.
[14] A. Samusenko, S. Esaulov, and V.A. Ivchin. Simulation of Helicopter Vibration Reduction by Means of Higher Harmonics Control with the Use of Flight Simulator. In 38th European Rotorcraft Forum, Amsterdam, Netherlands, Paper No13, September 4-6 2012. .

[15] B.W. Sim, M. Potsdam, C. Kitaplioglu, P. LeMasurier, P. Lorber, and J. Andrews. Localized, Non-Harmonic Active Flap Motions for Low Frequency In-Plane Noise Reduction. In American Helicopter Society 68th Annual Forum, Fort Worth, TX, May 1-3 2012.

[16] R. Steijl, G. Barakos, and K. Badcock. A framework for CFD analysis of helicopter rotors in hover and forward flight. International Journal for Numerical Methods in Fluids, 51(8):819-847, 2006.

[17] R. Steijl, M. Woodgate, and G. Barakos. CFD Requirements for Efficient Smart-Rotor Analysis. In 35th European Rotorcraft Forum, Hamburg, Germany, Paper No1187, September 16-18 2009.

[18] F.K. Straub, V.R. Anand, T.S. Birchette, and B.H. Lau. SMART Rotor Development and Wind Tunnel Test. In 35th European Rotorcraft Forum, Hamburg, Germany, Paper No1200, September 22-25 2009. .

[19] A. Tamer, V. Muscarello, P. Masarati, and G. Quaranta. Helicopter Vibratory Loads and Vibrations Reduction Using Higher-Harmonic Control. In 39th European Rotorcraft Forum, Moscow, Russia, Paper No37, September 3-5 2013. .

[20] G.D. van Albada, B. B. van Leer, and W.W. Roberts. A Comparative Study of Computational Methods in Cosmic Gas Dynamics. Astronomy and Astrophysics, 108(1):76-84, April 1982.

Table 1: Conditions for the pitching aerofoil case.

\begin{tabular}{|l|c|c|c|c|c|}
\hline \hline Sections & $M_{\infty}$ & $R e_{\infty}$ & $k=\frac{\omega c}{2 V_{\infty}}$ & $\alpha$ (degrees) & $\delta_{f}$ (degrees) \\
\hline \hline Inboard & 0.3 & $4.2 \times 10^{6}$ & 0.08 & $10+5 \sin (\psi)$ & $0,-2.5,4.5,3+3 \sin (\psi)$ \\
\hline Inboard & 0.3 & $4.2 \times 10^{6}$ & 0.08 & $10+10 \sin (\psi)$ & $0,-2.5,4.5,3+3 \sin (\psi)$ \\
\hline Inboard & 0.3 & $4.2 \times 10^{6}$ & 0.08 & $14+5 \sin (\psi)$ & $0,-2.5,4.5,3+3 \sin (\psi)$ \\
\hline Inboard & 0.3 & $4.2 \times 10^{6}$ & 0.08 & $14+14 \sin (\psi)$ & $0,-2.5,4.5,3+3 \sin (\psi)$ \\
\hline Inboard & 0.3 & $4.2 \times 10^{6}$ & 0.12 & $10+5 \sin (\psi)$ & $0,-2.5,4.5$ \\
\hline Inboard & 0.3 & $4.2 \times 10^{6}$ & 0.12 & $10+10 \sin (\psi)$ & $0,-2.5,4.5$ \\
\hline Inboard & 0.3 & $4.2 \times 10^{6}$ & 0.12 & $14+5 \sin (\psi)$ & $0,-2.5,4.5$ \\
\hline Inboard & 0.3 & $4.2 \times 10^{6}$ & 0.12 & $14+14 \sin (\psi)$ & $0,-2.5,4.5$ \\
\hline \hline Outboard & 0.3 & $4.2 \times 10^{6}$ & 0.08 & $10+5 \sin (\psi)$ & $0,-2.5,4.5$ \\
\hline Outboard & 0.3 & $4.2 \times 10^{6}$ & 0.08 & $10+10 \sin (\psi)$ & $0,-2.5,4.5$ \\
\hline Outboard & 0.3 & $4.2 \times 10^{6}$ & 0.08 & $14+5 \sin (\psi)$ & $0,-2.5,4.5$ \\
\hline Outboard & 0.3 & $4.2 \times 10^{6}$ & 0.08 & $14+14 \sin (\psi)$ & $0,-2.5,4.5$ \\
\hline Outboard & 0.3 & $4.2 \times 10^{6}$ & 0.12 & $10+5 \sin (\psi)$ & $0,-2.5,4.5$ \\
\hline Outboard & 0.3 & $4.2 \times 10^{6}$ & 0.12 & $10+10 \sin (\psi)$ & $0,-2.5,4.5$ \\
\hline Outboard & 0.3 & $4.2 \times 10^{6}$ & 0.12 & $14+5 \sin (\psi)$ & $0,-2.5,4.5$ \\
\hline Outboard & 0.3 & $4.2 \times 10^{6}$ & 0.12 & $14+14 \sin (\psi)$ & $0,-2.5,4.5$ \\
\hline \hline
\end{tabular}


Table 2: Conditions for the dMdt Cases

\begin{tabular}{|l|c|c|c|c|c|}
\hline \hline Case & Section & $M_{\infty}$ & $R e_{\infty}$ & $\alpha$ (degrees) & $\delta_{f}$ (degrees) \\
\hline \hline 1 & Inboard & $0.387+0.24 \sin (\psi)$ & $5.418 \times 10^{6}$ & $7-5 \sin (\psi)$ & 0 \\
\hline 2 & Inboard & $0.387+0.24 \sin (\psi)$ & $5.418 \times 10^{6}$ & $7-5 \sin (\psi)$ & -2.5 \\
\hline 3 & Inboard & $0.387+0.24 \sin (\psi)$ & $5.418 \times 10^{6}$ & $7-5 \sin (\psi)$ & 4.5 \\
\hline 4 & Inboard & $0.387+0.24 \sin (\psi)$ & $5.418 \times 10^{6}$ & $7-5 \sin (\psi)$ & $1-3.5 \sin (\psi)$ \\
\hline \hline 5 & Outboard & $0.5+0.24 \sin (\psi)$ & $7.0 \times 10^{6}$ & $5-5 \sin (\psi)$ & 0 \\
\hline 6 & Outboard & $0.5+0.24 \sin (\psi)$ & $7.0 \times 10^{6}$ & $5-5 \sin (\psi)$ & -2.5 \\
\hline 7 & Outboard & $0.5+0.24 \sin (\psi)$ & $7.0 \times 10^{6}$ & $5-5 \sin (\psi)$ & 4.5 \\
\hline 8 & Outboard & $0.5+0.24 \sin (\psi)$ & $7.0 \times 10^{6}$ & $5-5 \sin (\psi)$ & $1-3.5 \sin (\psi)$ \\
\hline \hline
\end{tabular}

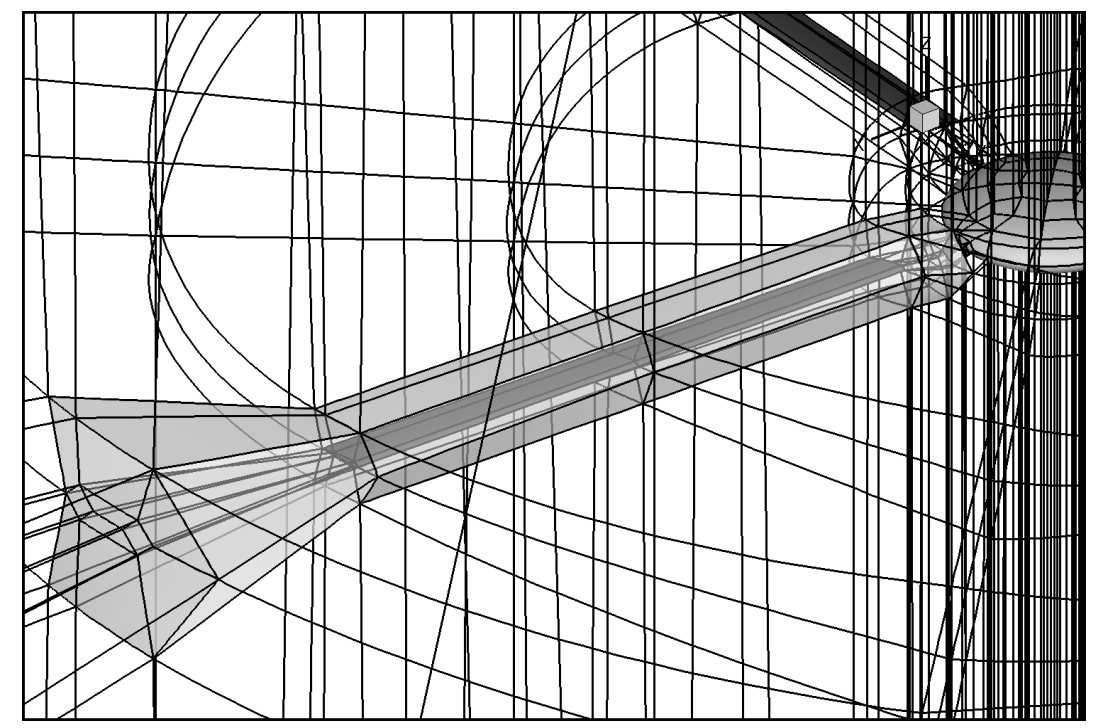

Figure 1: Rigid blocks (light grey) used for rotor trimming in HMB, for forward flight configurations. The blade is shown in dark grey and the hub in light grey. 


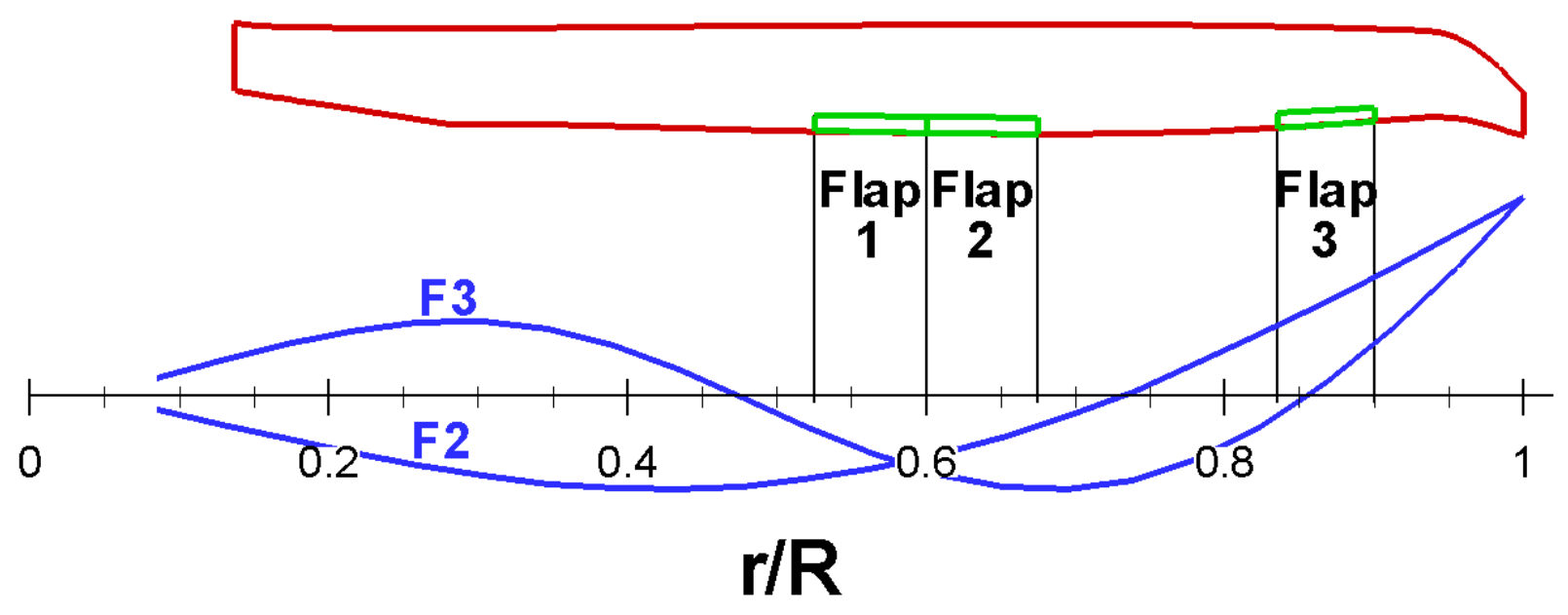

Figure 2: Location of the flaps on the blade compared to the second and third flapping mode shapes.

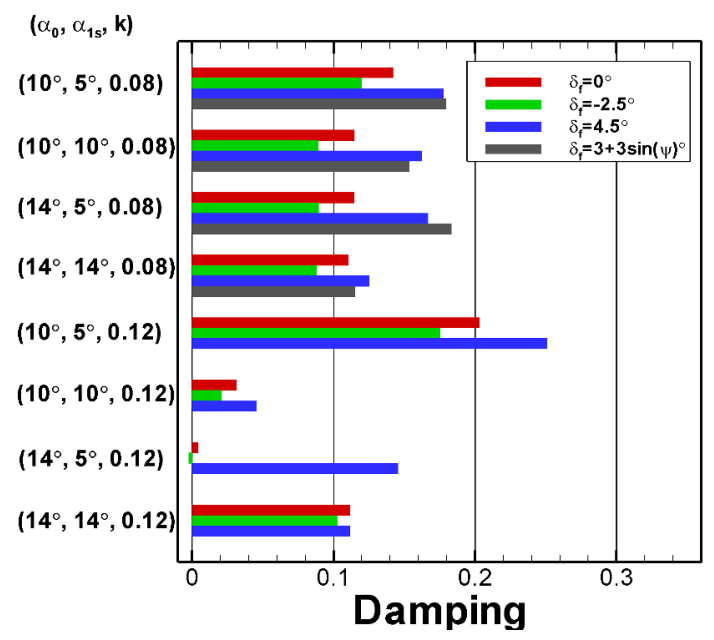

(a) Inboard section

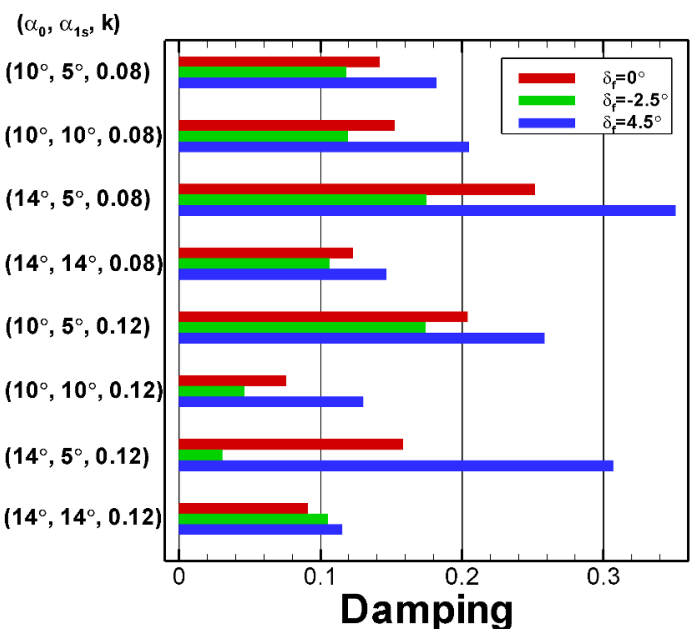

(b) Ouboard section

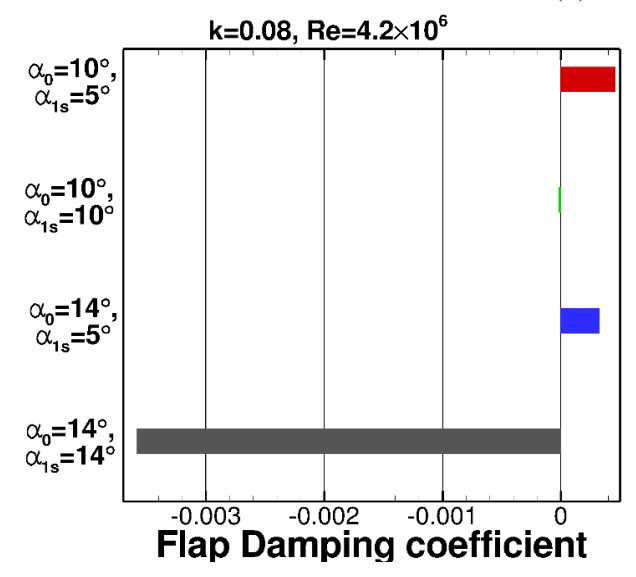

(c) Flap damping

Figure 3: Aerodynamic damping coefficient for pitching inboard and outboard sections, and aerodynamic flap damping when they are actuated. 


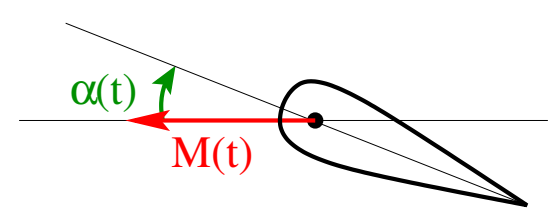

Figure 4: Aerofoil motion in a dMdt simulations.

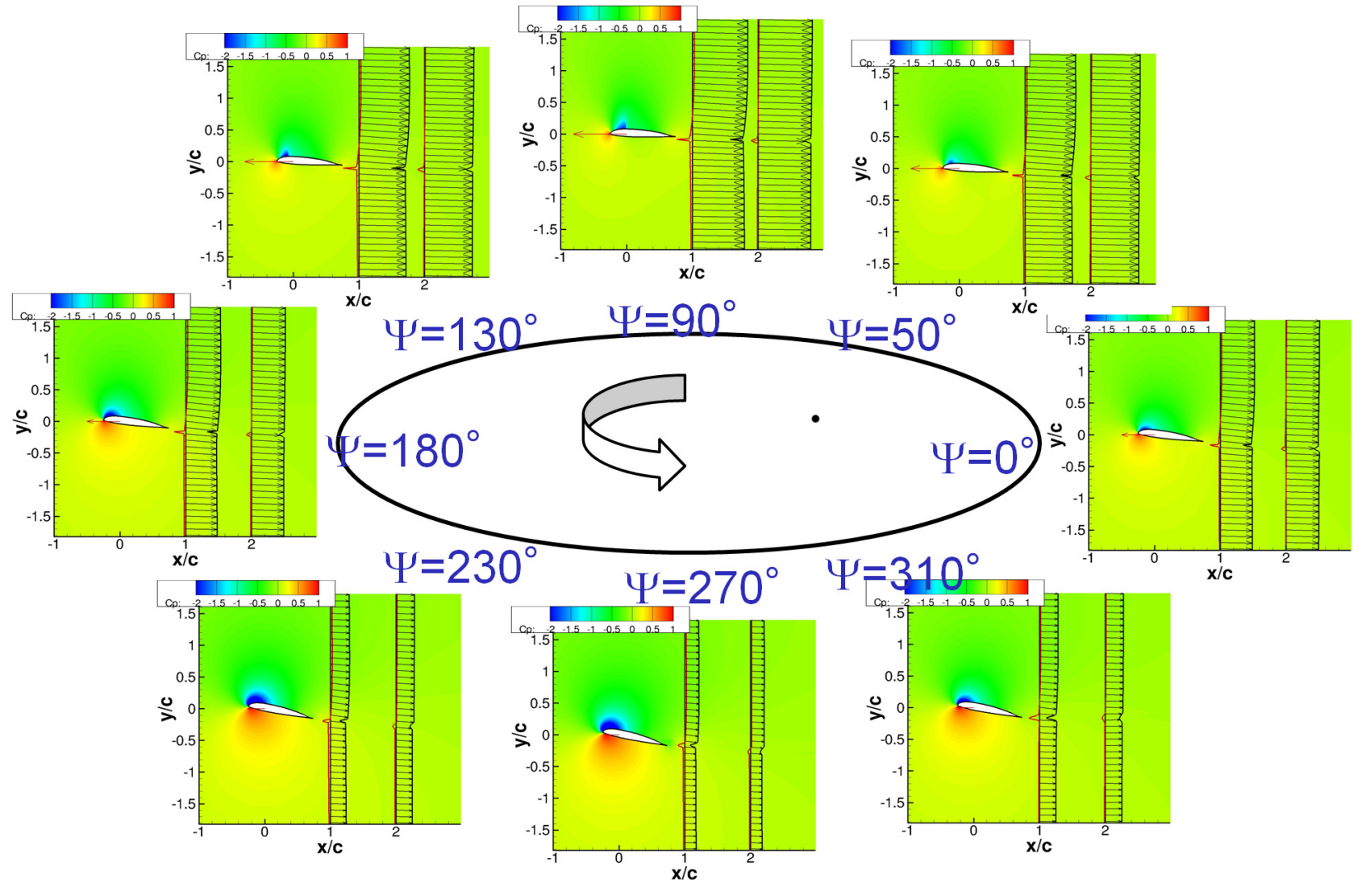

Figure 5: Evolution of the pressure coefficient around the inboard section and velocity distribution along two lines in the wake, Case 4. The black line shows velocities in the aerofoil frame of reference, and the red one shows velocities in the ground frame of reference. 


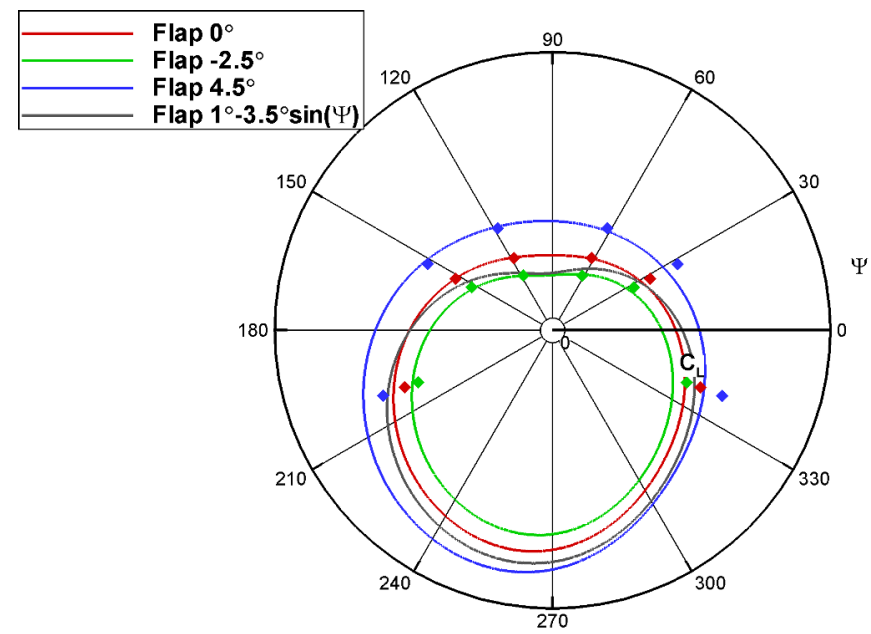

(a) $C_{L}$

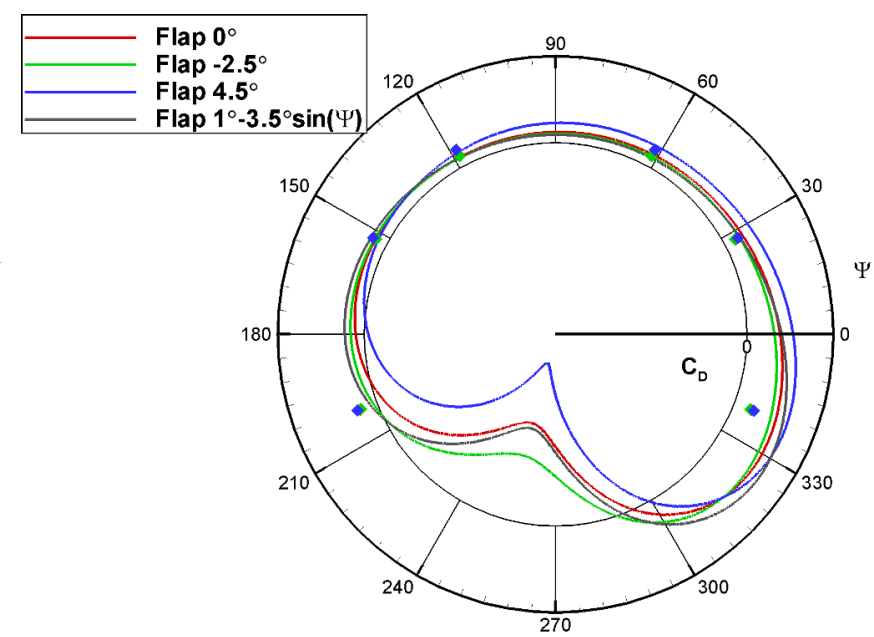

(b) $C_{D}$

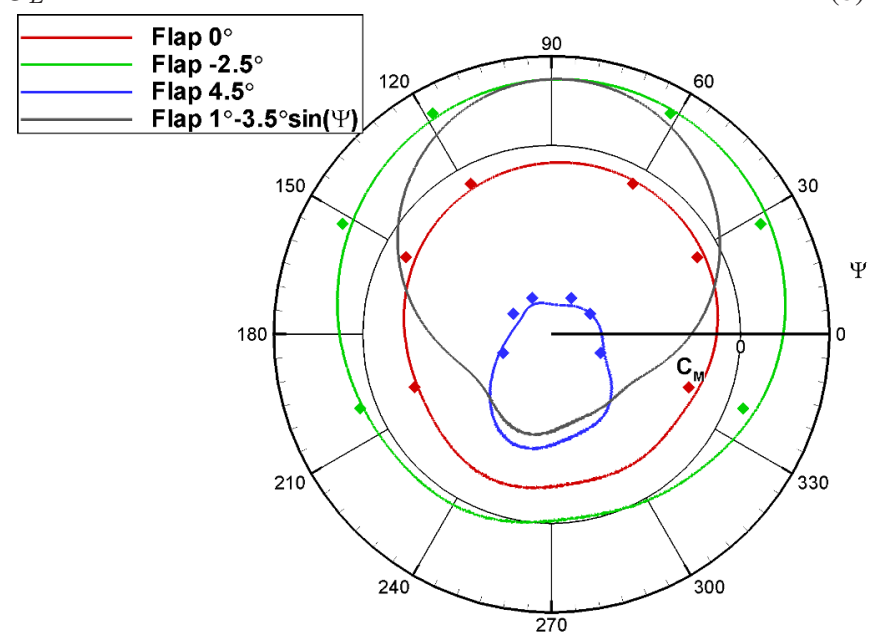

(c) $C_{M}$

Figure 6: Evolution of the lift, drag and moment coefficients with the azimuth for a dMdt simulation, Cases 1-4. The symbols show the force and moment coefficients obtained when using static simulations at similar conditions. 


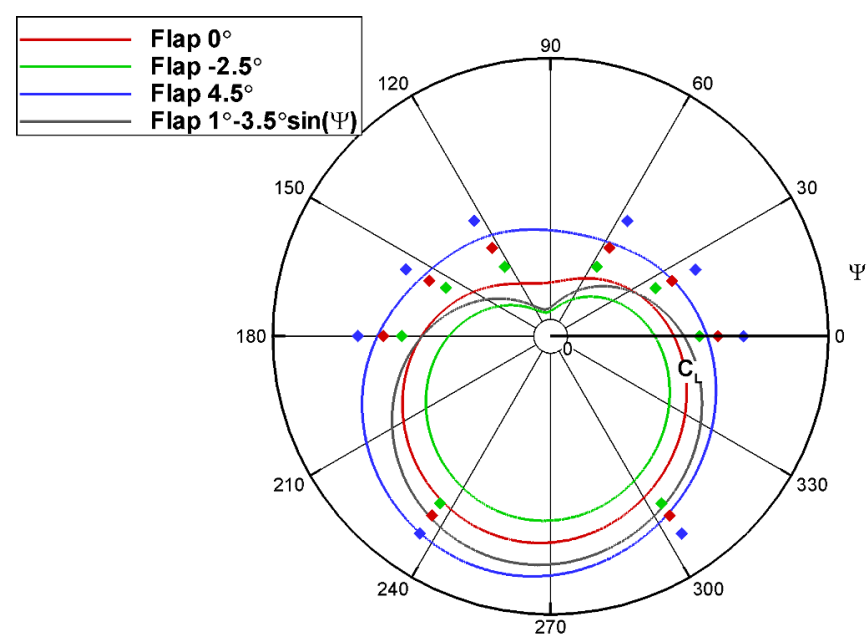

(a) $C_{L}$

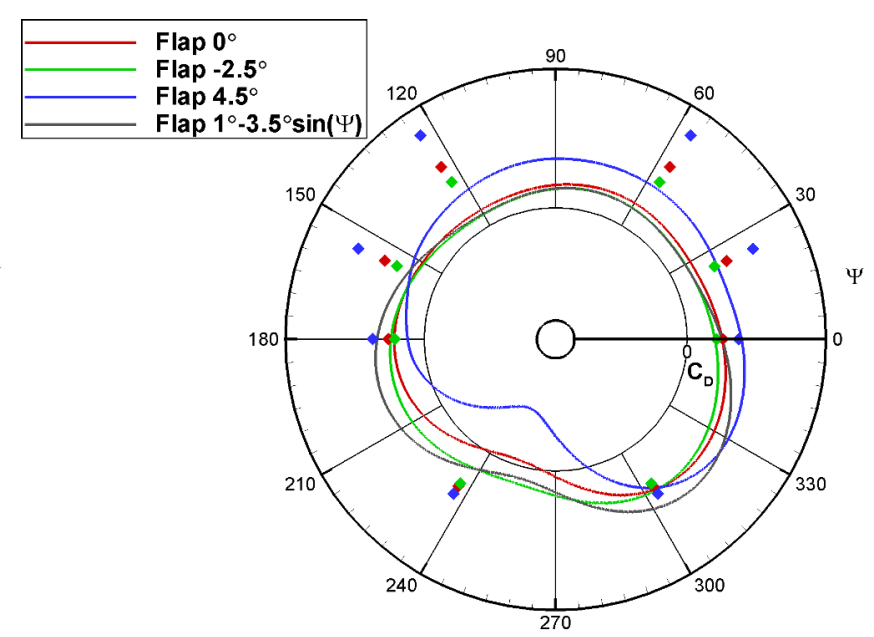

(b) $C_{D}$

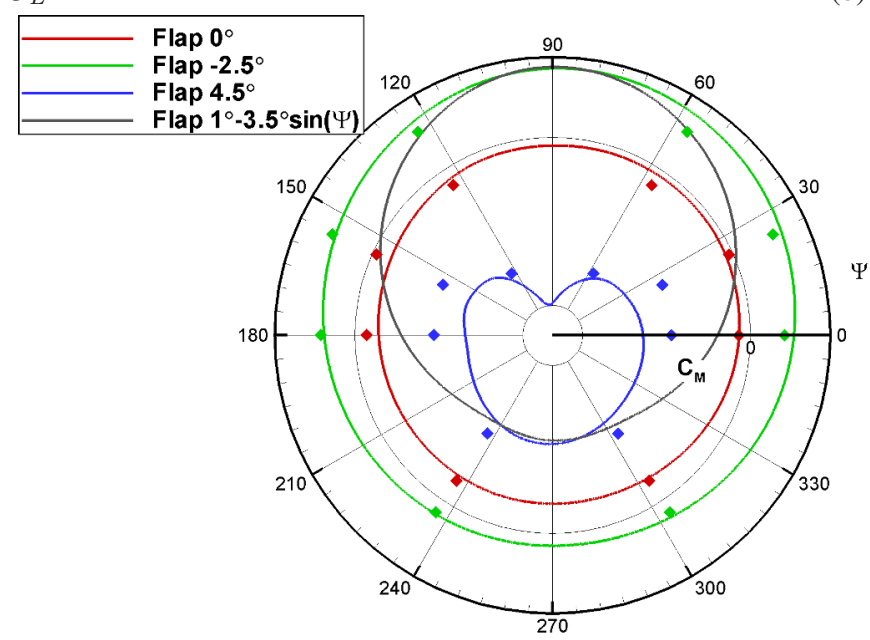

(c) $C_{M}$

Figure 7: Evolution of the lift, drag and moment coefficients with the azimuth for a dMdt simulation, Cases 5-8. The symbols show the force and moment coefficients obtained when using static simulations at similar conditions.

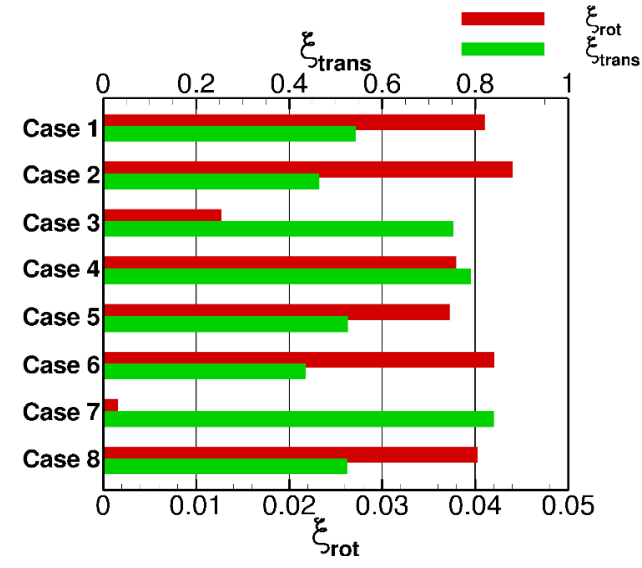

(a) Aerofoil damping coefficients

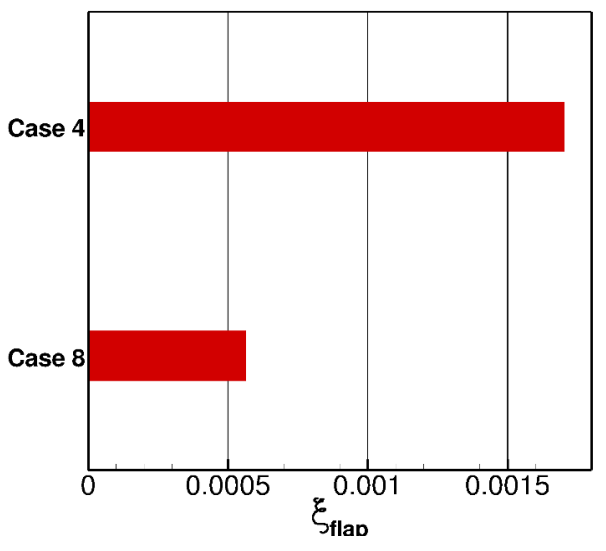

(b) Flap damping coefficient

Figure 8: Damping coefficient of the aerofoil in translation and pitching, and damping coefficient of the flap deployment when available. 


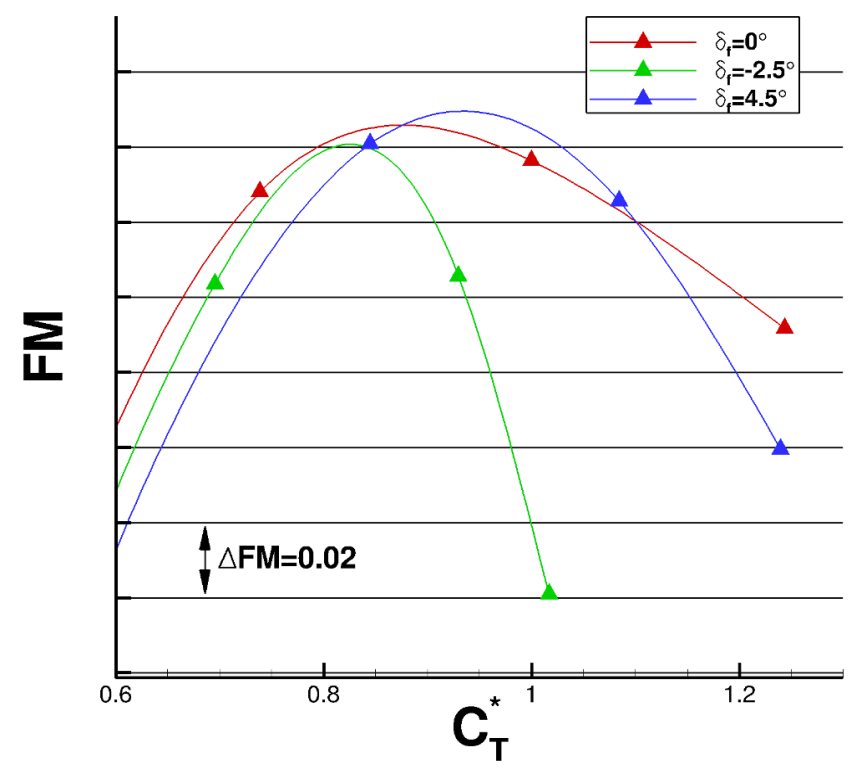

Figure 9: Evolution of the Figure of Merit with the flap deployment in hover.

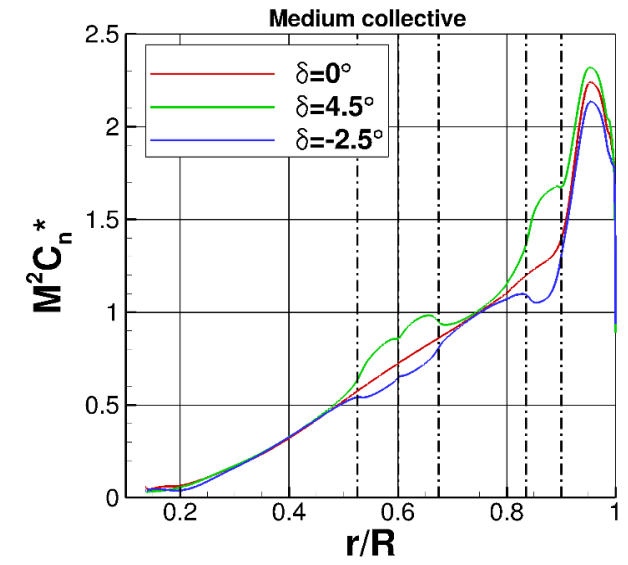

(a) Mach-scaled normal force coefficient

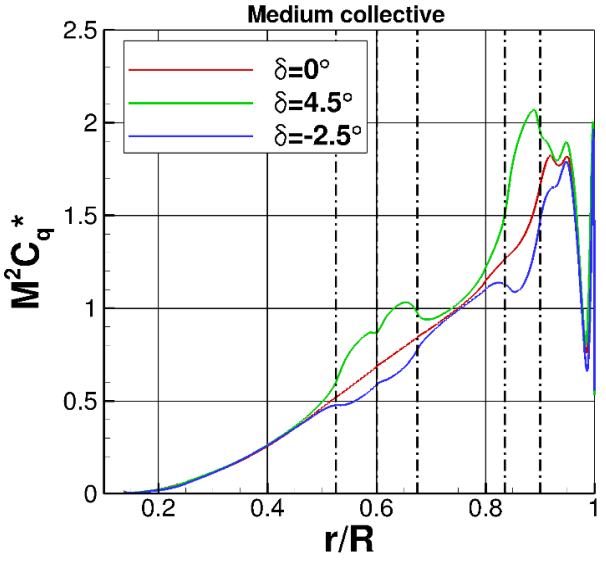

(b) Mach-scaled torque coefficient

Figure 10: Evolution of the Mach-scaled normal force and torque coefficients predictions with the flap deployment for a hovering main rotor blade, using a medium collective level. The coefficients were non-dimensionalised with their value at $r / R=0.75$ in the case without flap. 


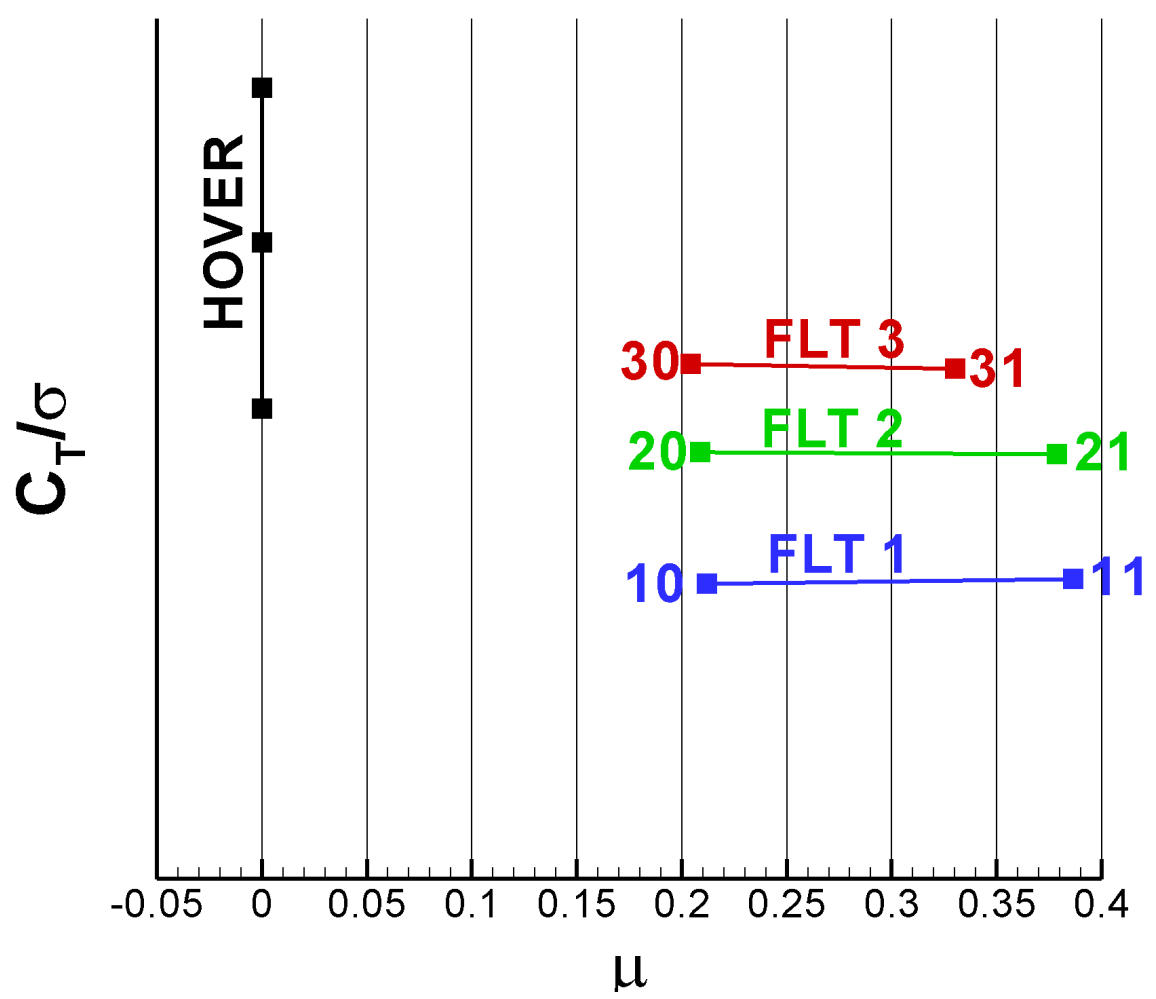

Figure 11: Flight conditions for the forward flight simulations. The location of the hover simulations performed without flaps are added for reference. 


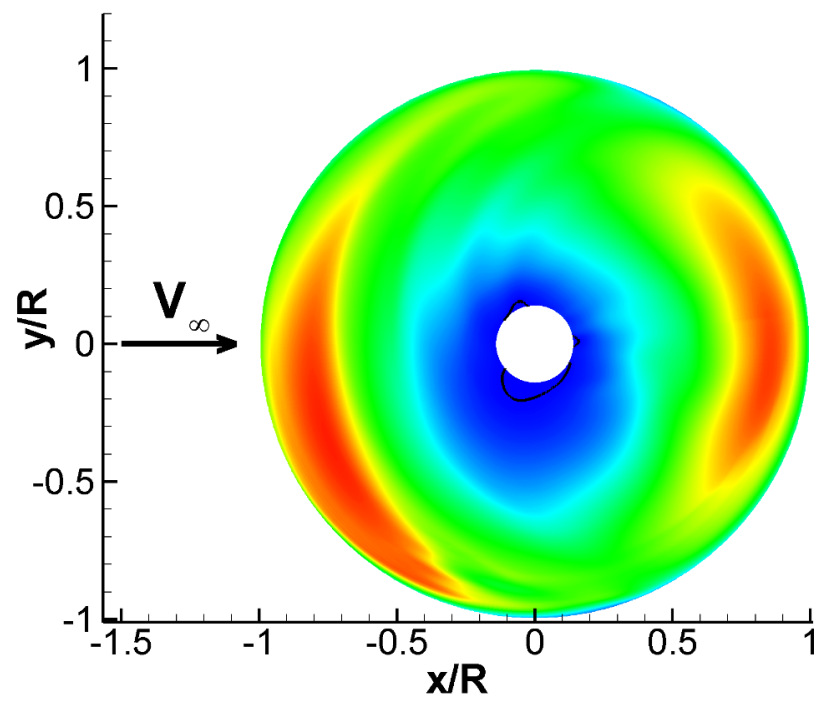

(a) $M^{2} C_{n}$, without flaps

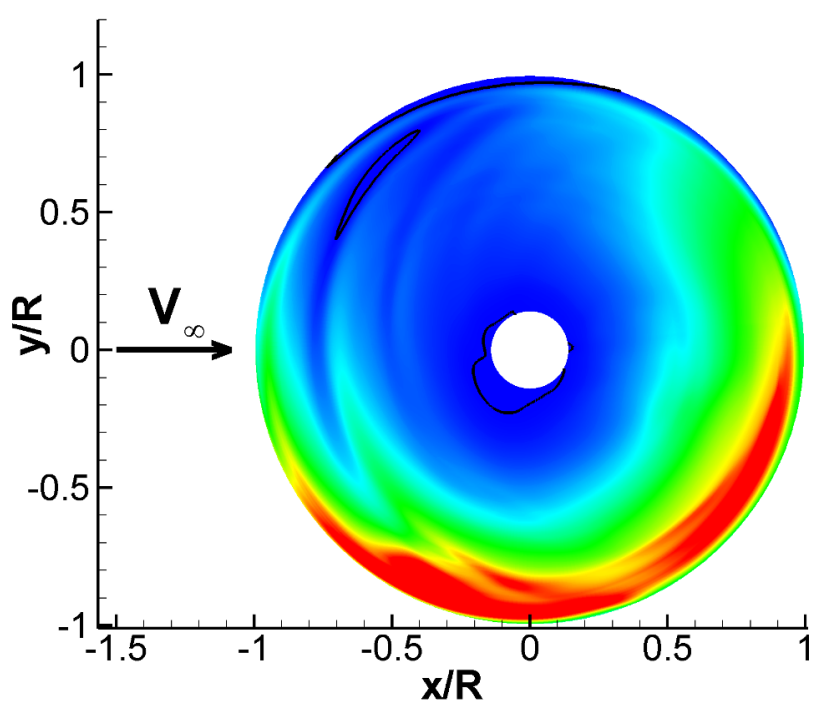

(c) $M^{2} C_{q}$, without flaps

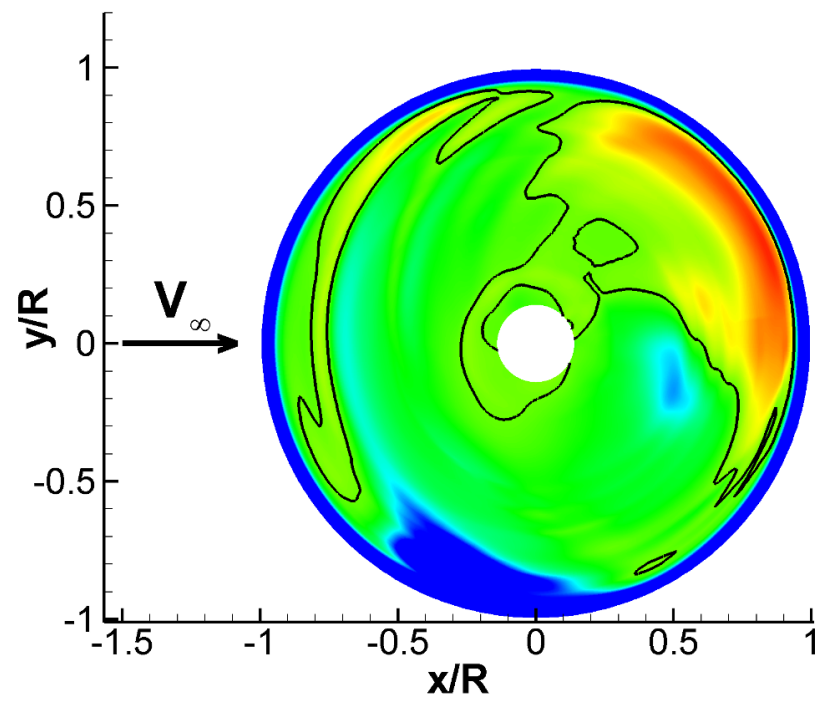

(e) $M^{2} C_{m}$, without flaps

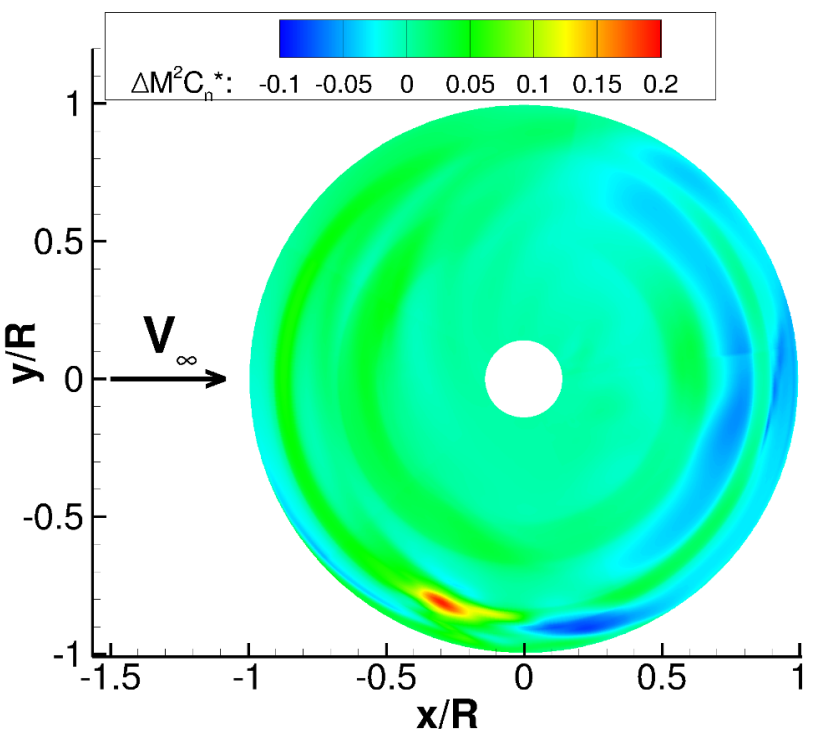

(b) $\Delta M^{2} C_{n}^{*}$

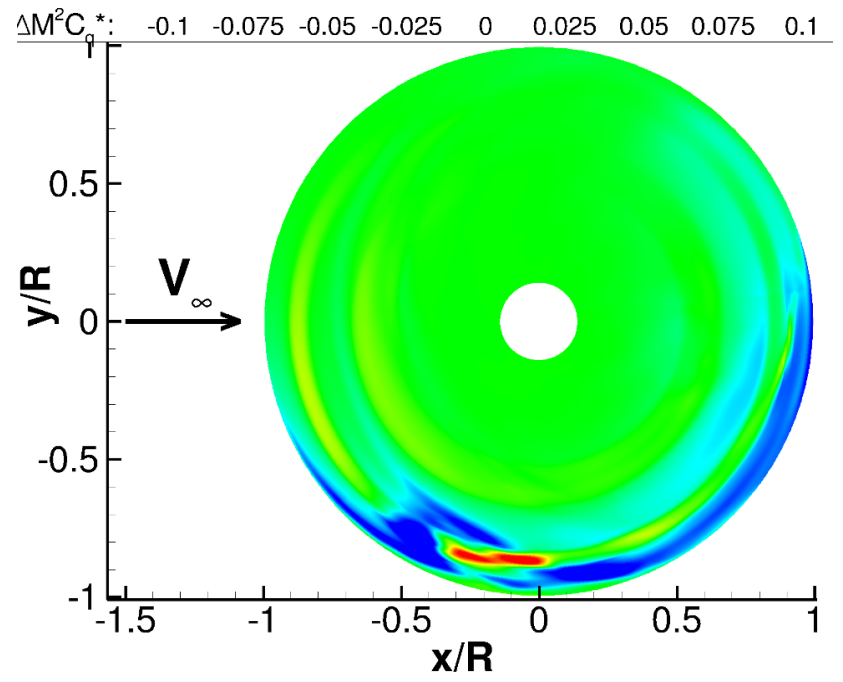

(d) $\Delta M^{2} C_{q}^{*}$

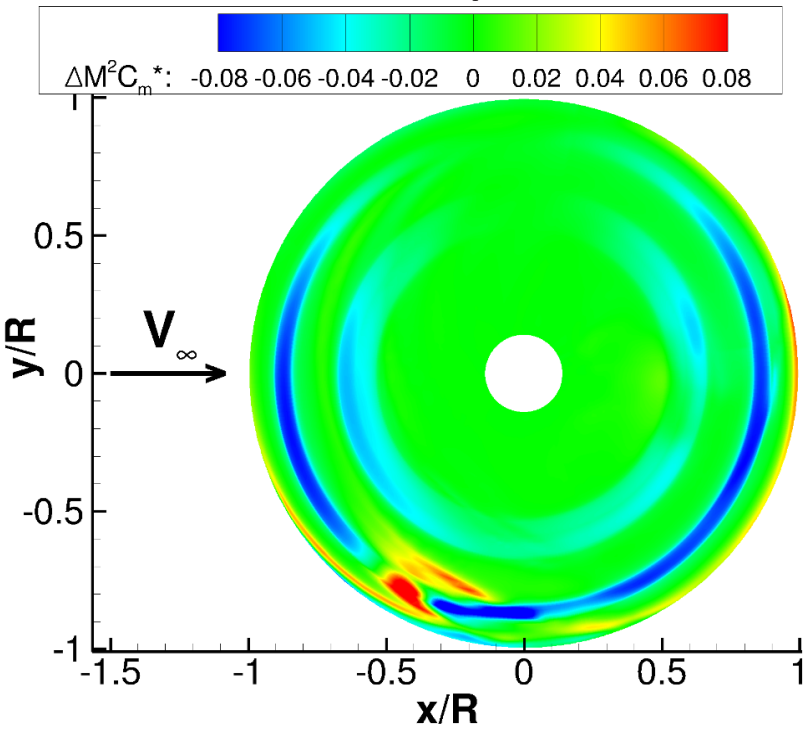

(f) $\Delta M^{2} C_{m}^{*}$

Figure 12: Mach-scaled normal force, torque and moment coefficient predictions for Flight 3-30. In the plots without flaps, a thick black lines indicates a value of 0 . 


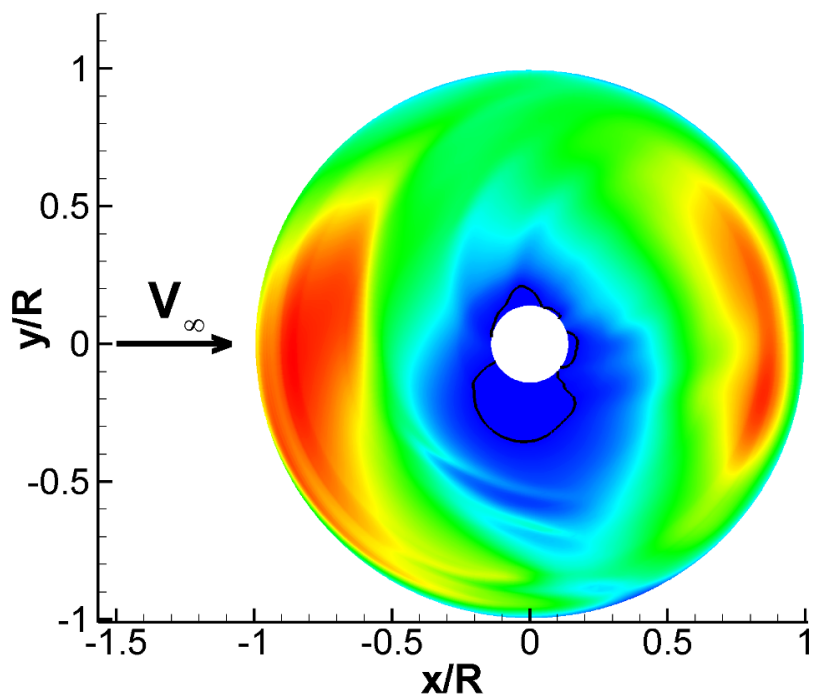

(a) $M^{2} C_{n}$, without flaps

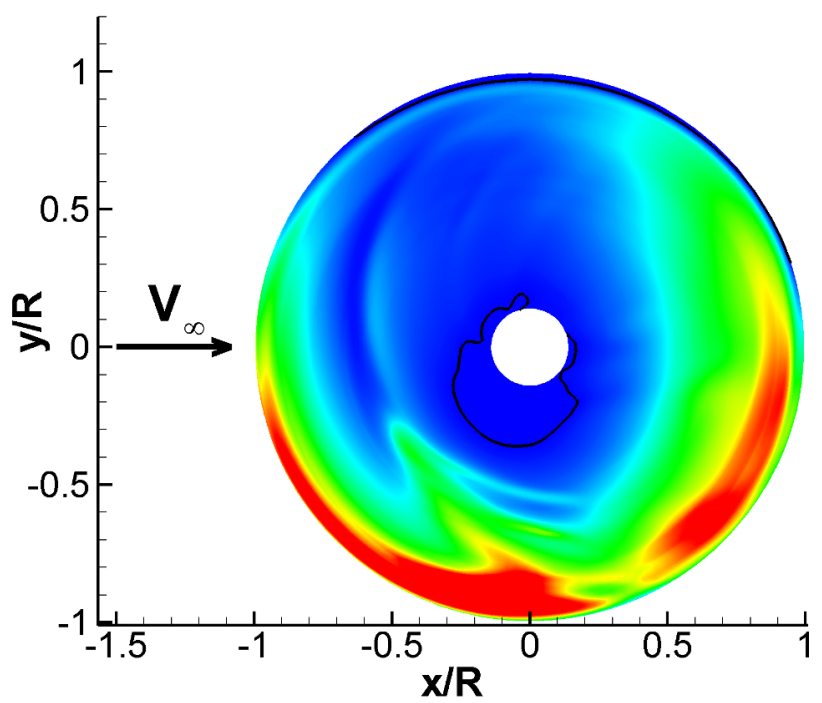

(c) $M^{2} C_{q}$, without flaps

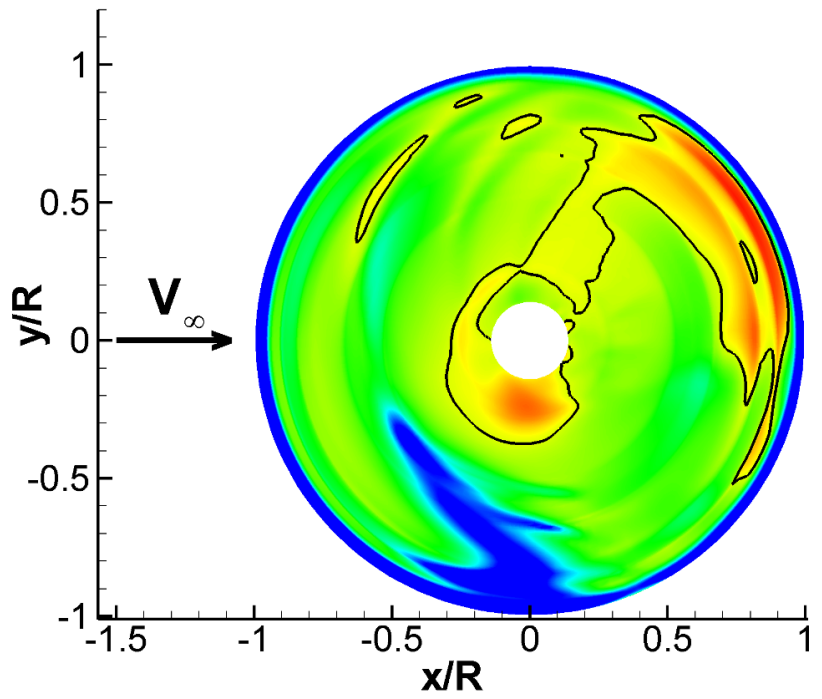

(e) $M^{2} C_{m}$, without flaps

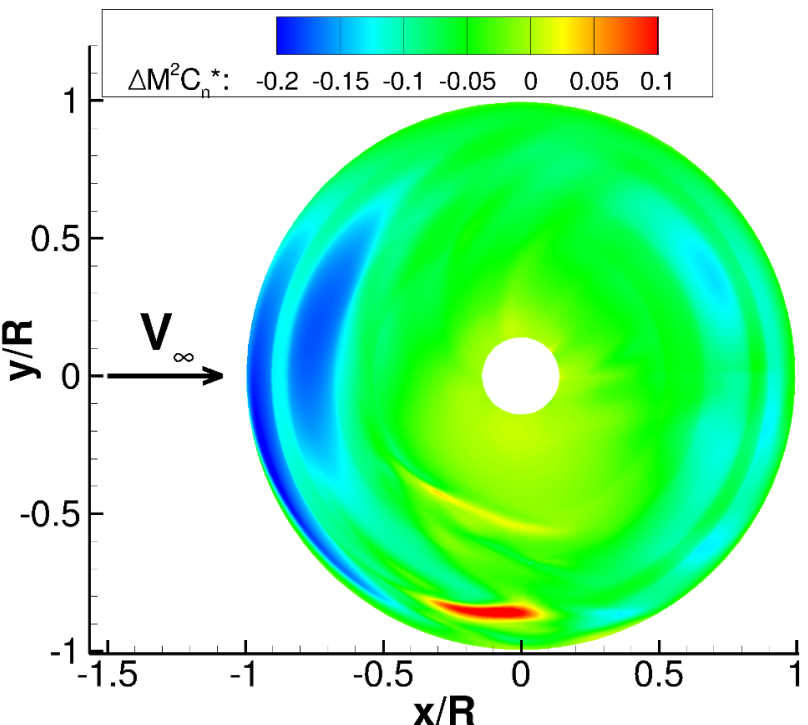

(b) $\Delta M^{2} C_{n}^{*}$

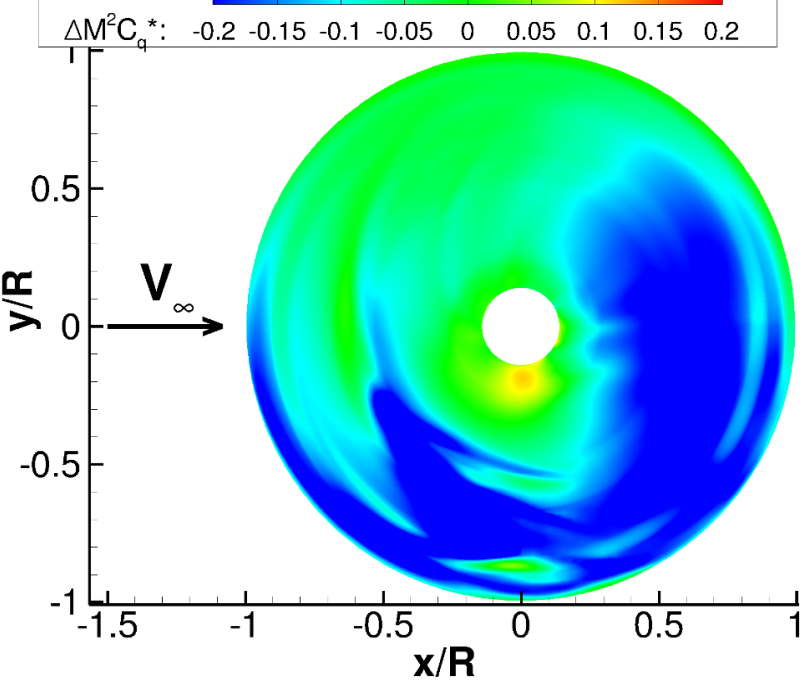

(d) $\Delta M^{2} C_{q}^{*}$

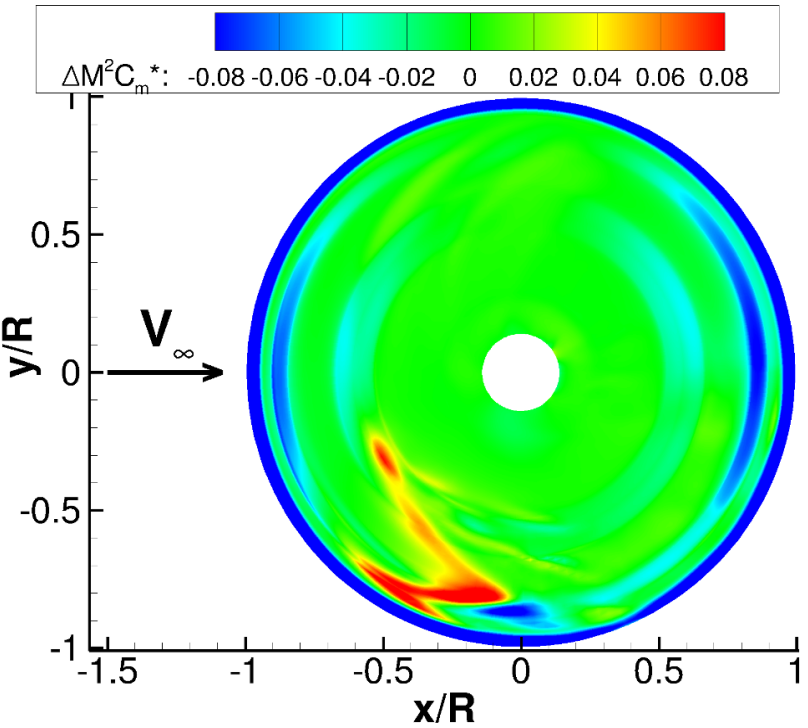

(f) $\Delta M^{2} C_{m}^{*}$

Figure 13: Mach-scaled normal force, torque and moment coefficient predictions for Flight 3-31. In the plots without flaps, thick black lines indicate a value of 0 . 


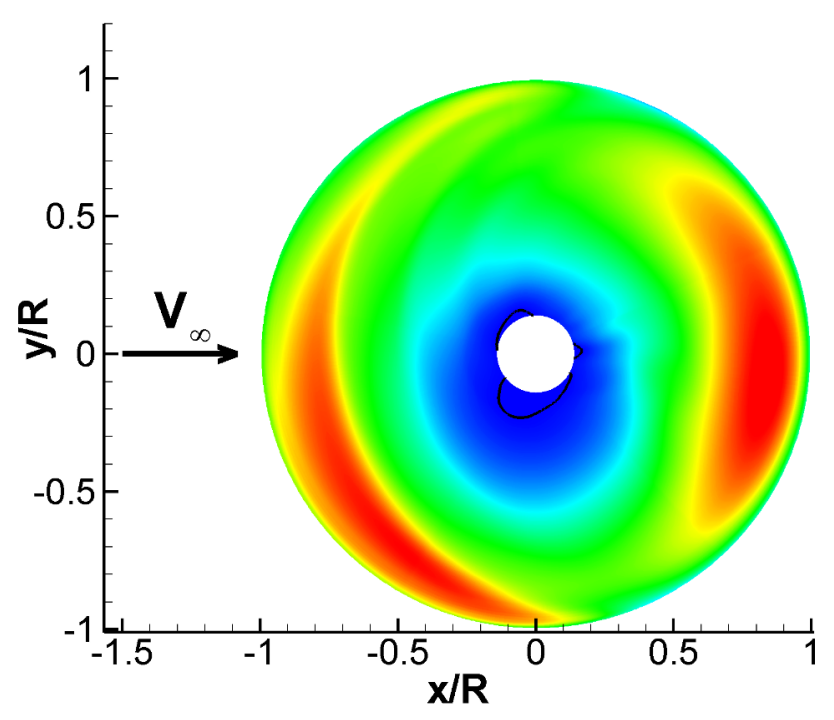

(a) $M^{2} C_{n}$, without flaps

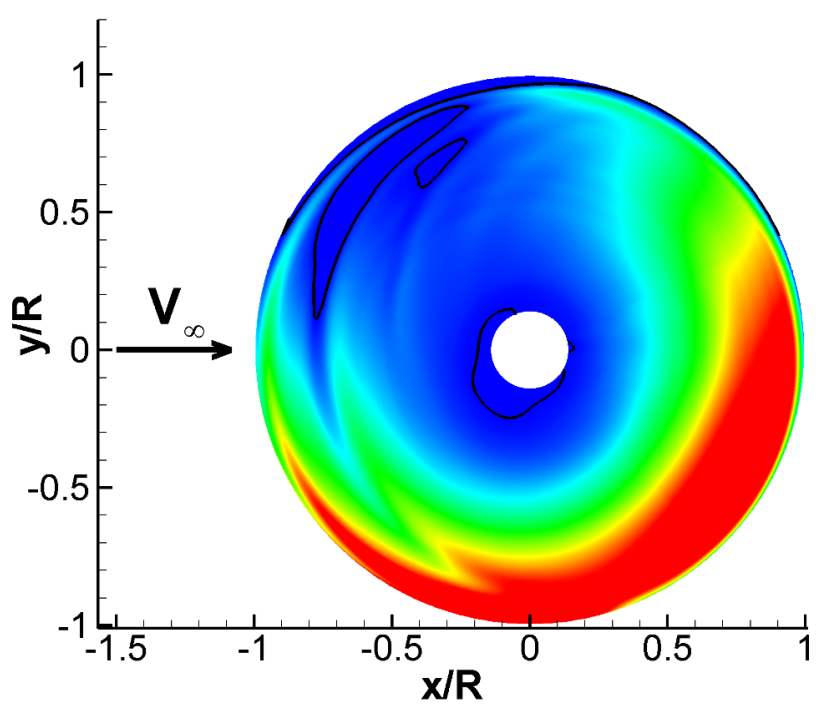

(c) $M^{2} C_{q}$, without flaps

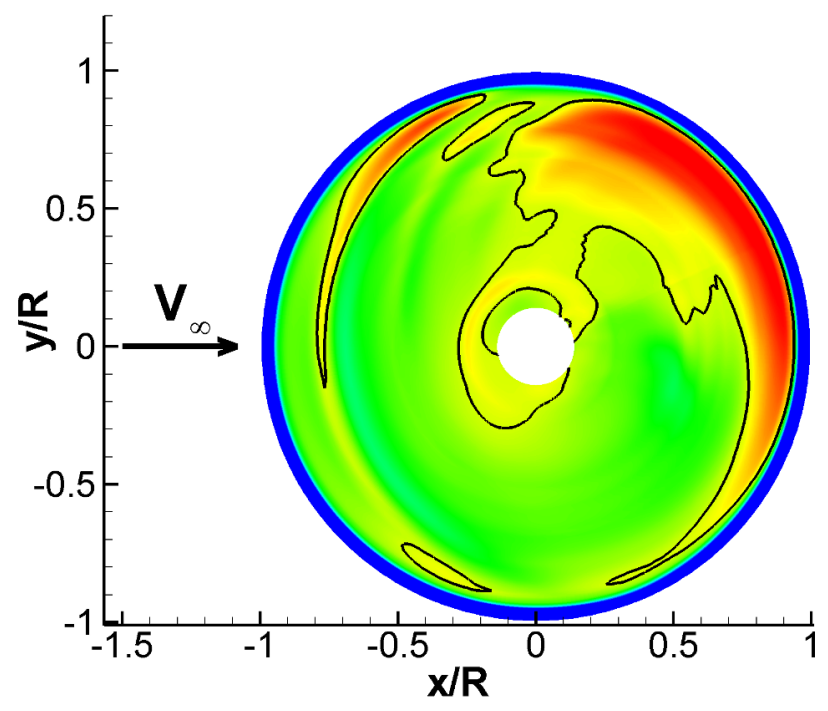

(e) $M^{2} C_{m}$, without flaps

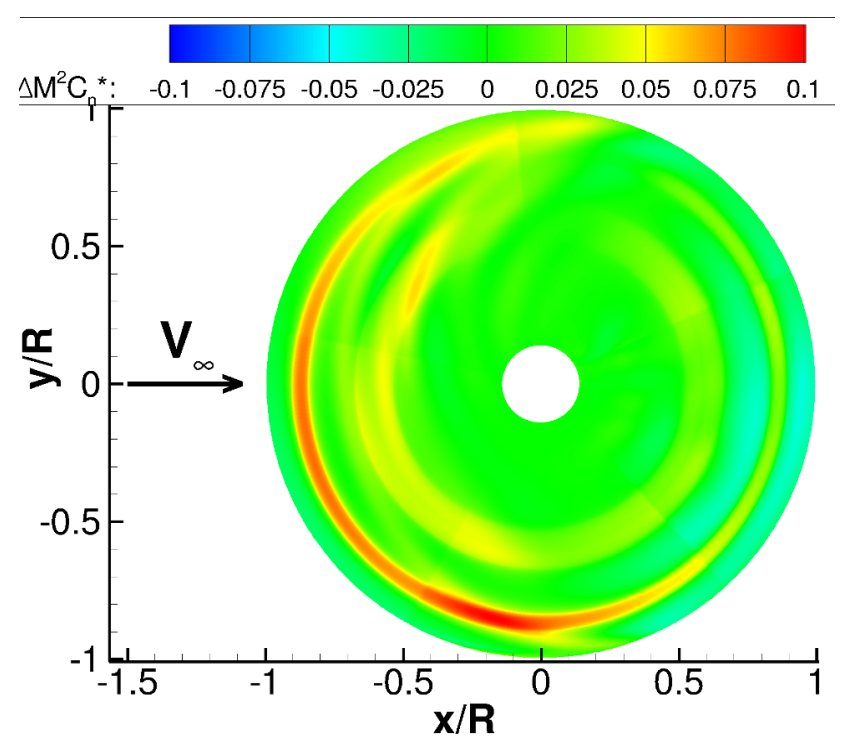

(b) $\Delta M^{2} C_{n}^{*}$

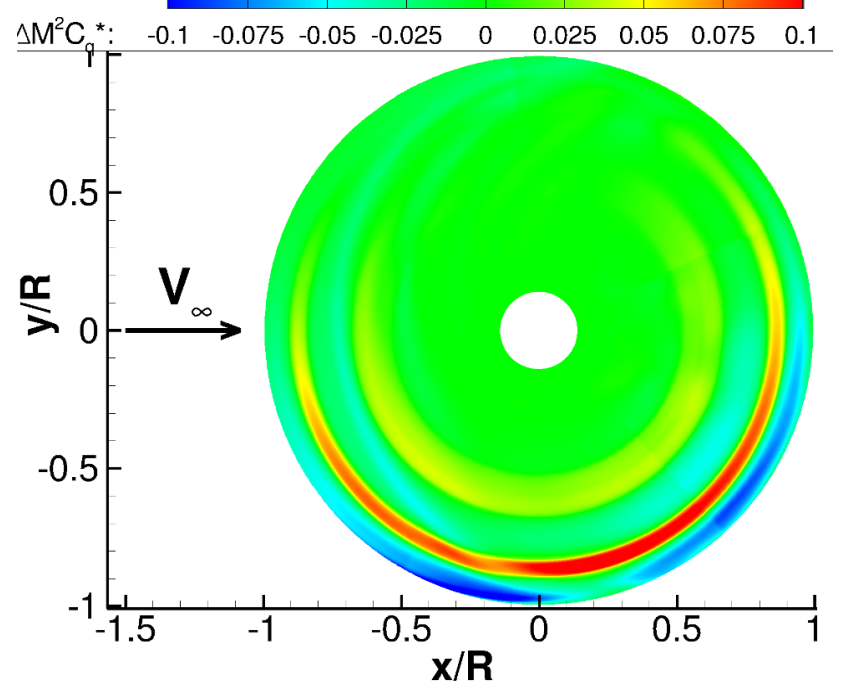

(d) $\Delta M^{2} C_{q}^{*}$

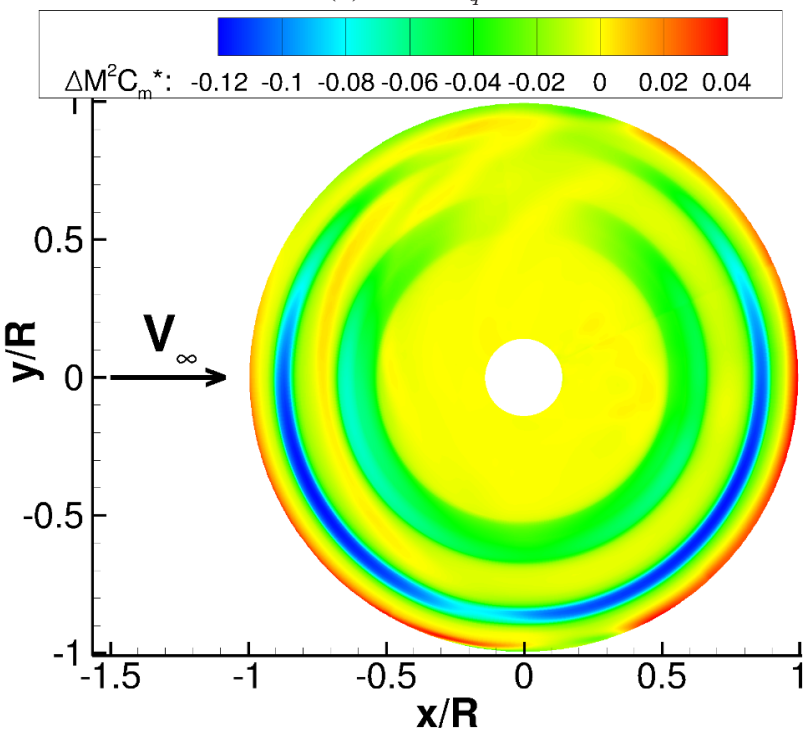

(f) $\Delta M^{2} C_{m}^{*}$

Figure 14: Mach-scaled normal force, torque and moment coefficient predictions for Flight 2-20. In the plots without flaps, thick black lines indicate a value of 0 . 


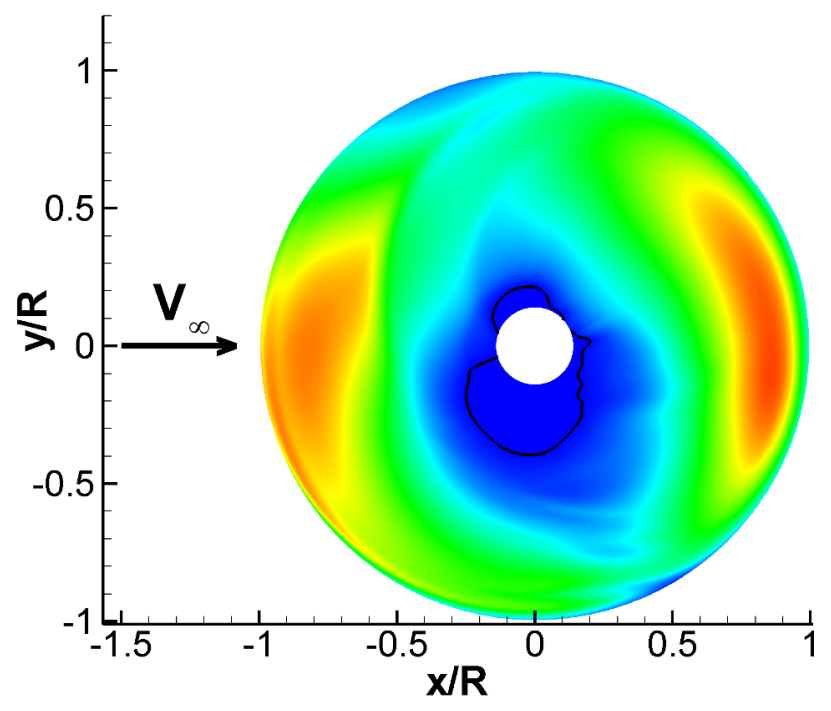

(a) $M^{2} C_{n}$, without flaps

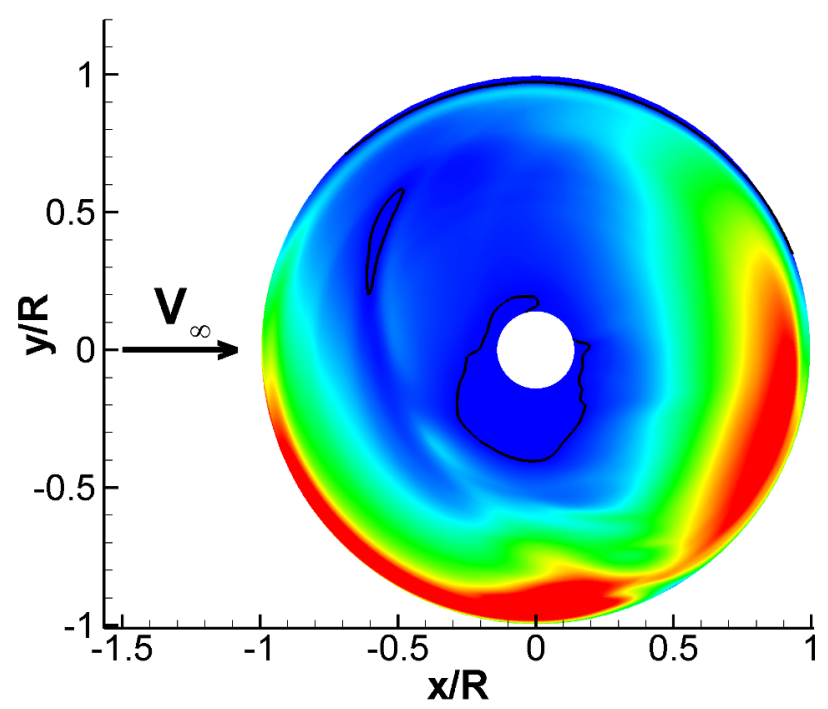

(c) $M^{2} C_{q}$, without flaps

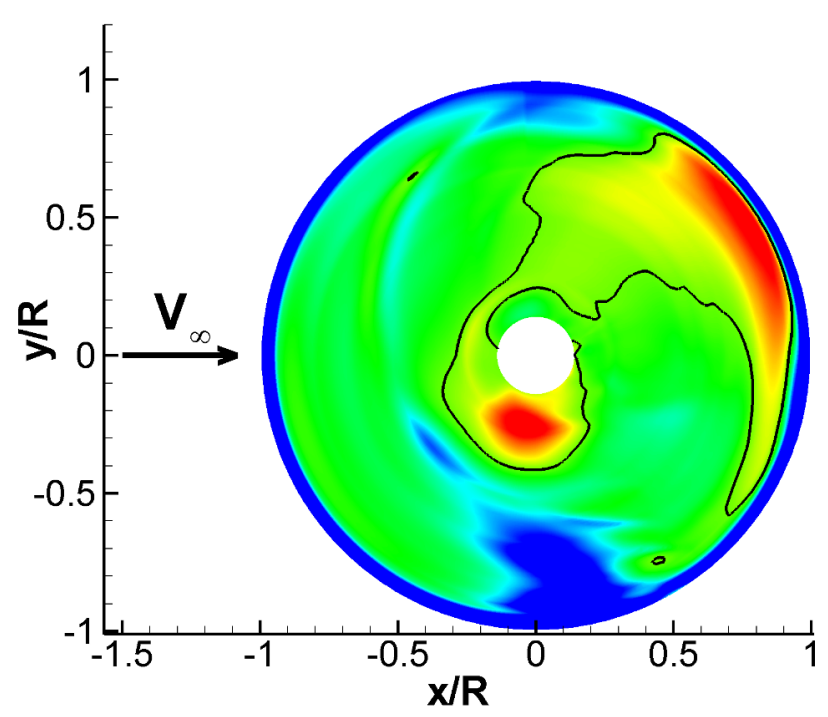

(e) $M^{2} C_{m}$, without flaps

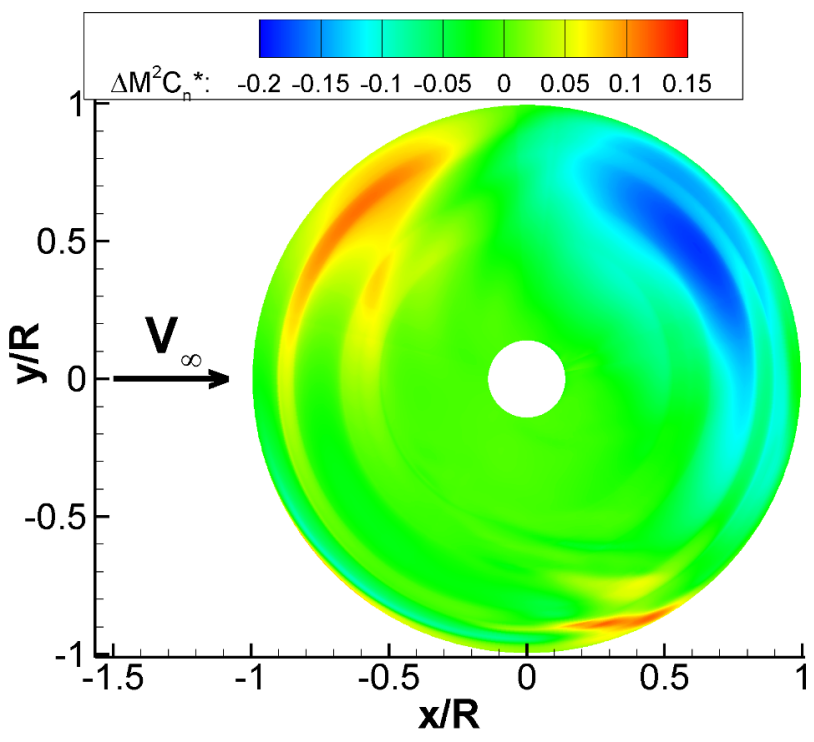

(b) $\Delta M^{2} C_{n}^{*}$

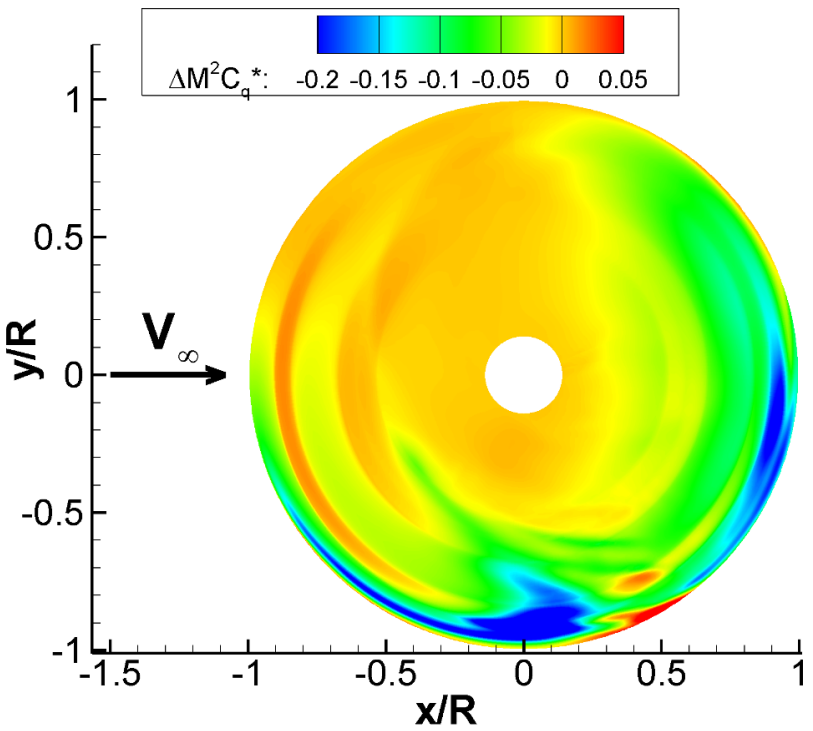

(d) $\Delta M^{2} C_{q}^{*}$

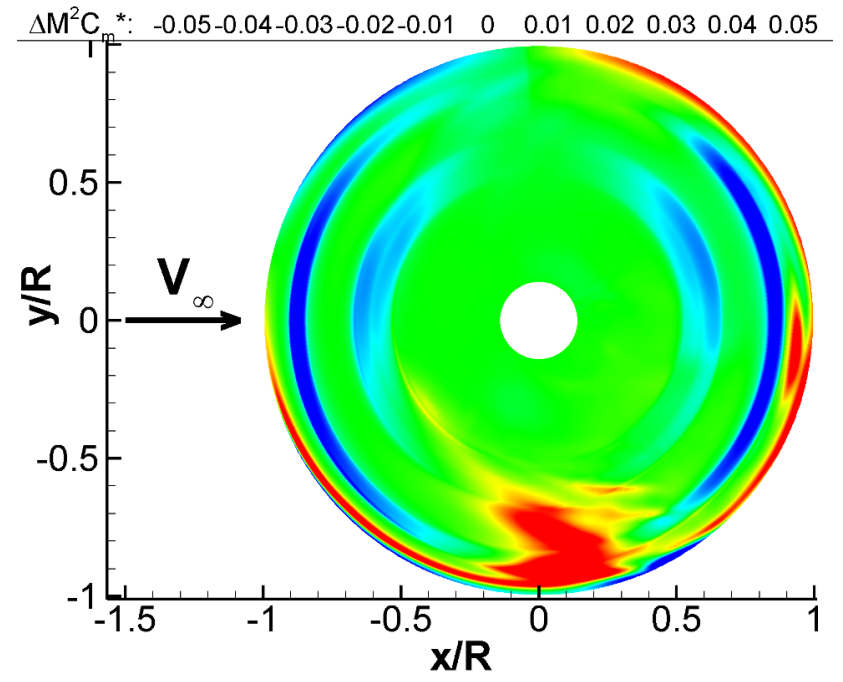

(f) $\Delta M^{2} C_{m}^{*}$

Figure 15: Mach-scaled normal force, torque and moment coefficient predictions for Flight 2-21. In the plots without flaps, thick black lines indicate a value of 0 . 


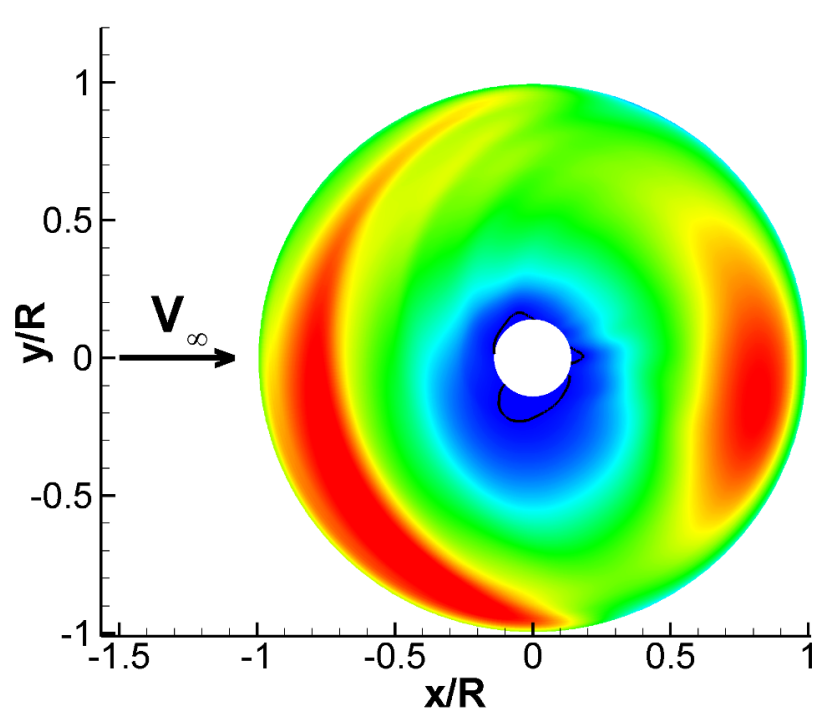

(a) $M^{2} C_{n}$, without flaps

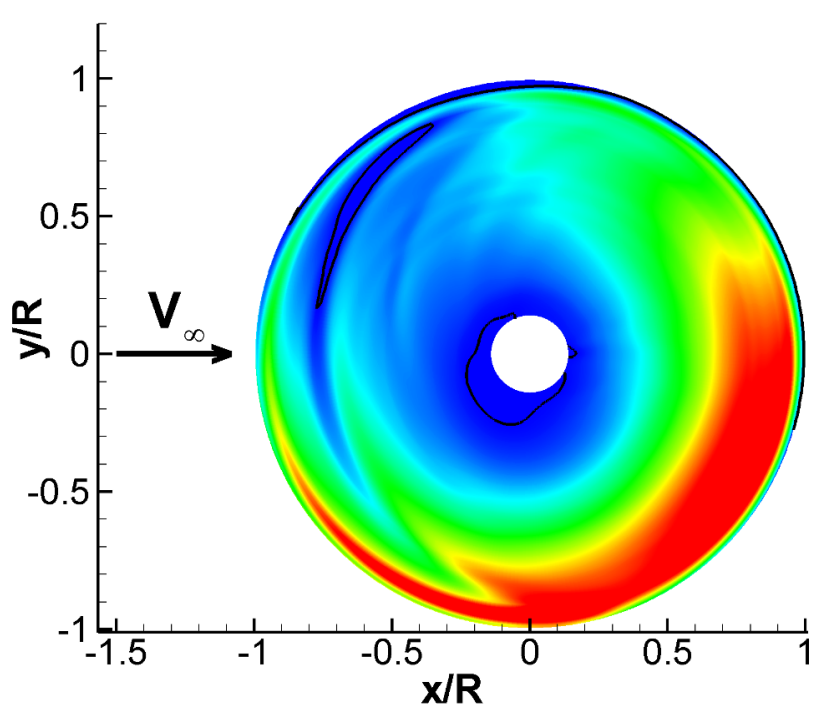

(c) $M^{2} C_{q}$, without flaps

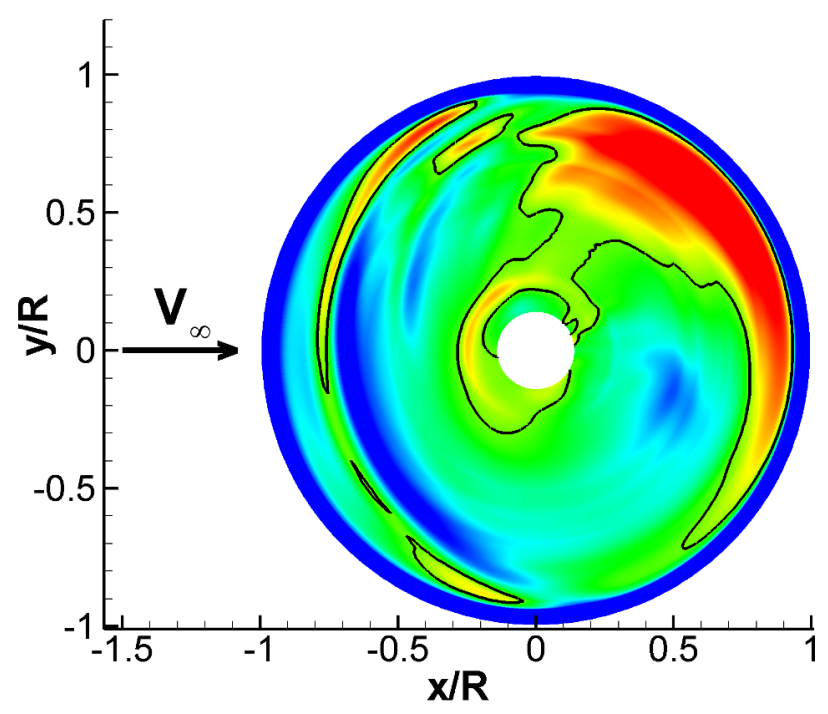

(e) $M^{2} C_{m}$, without flaps

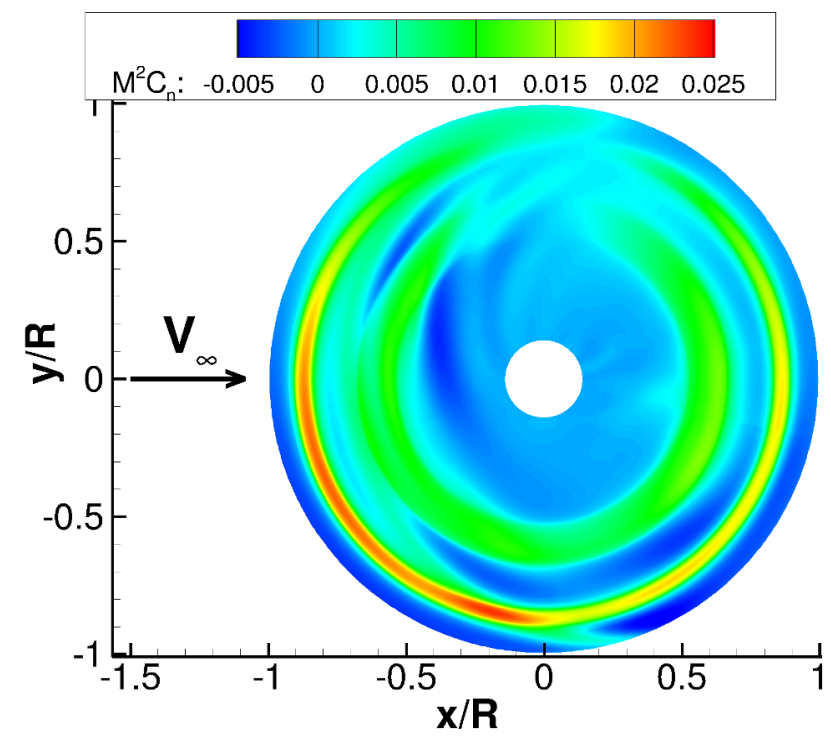

(b) $\Delta M^{2} C_{n}^{*}$

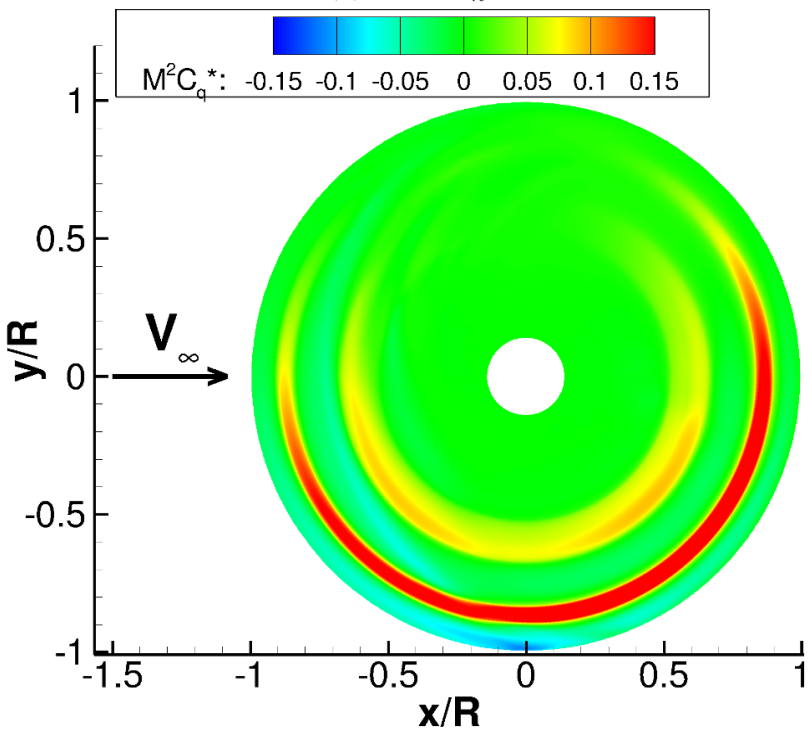

(d) $\Delta M^{2} C_{q}^{*}$

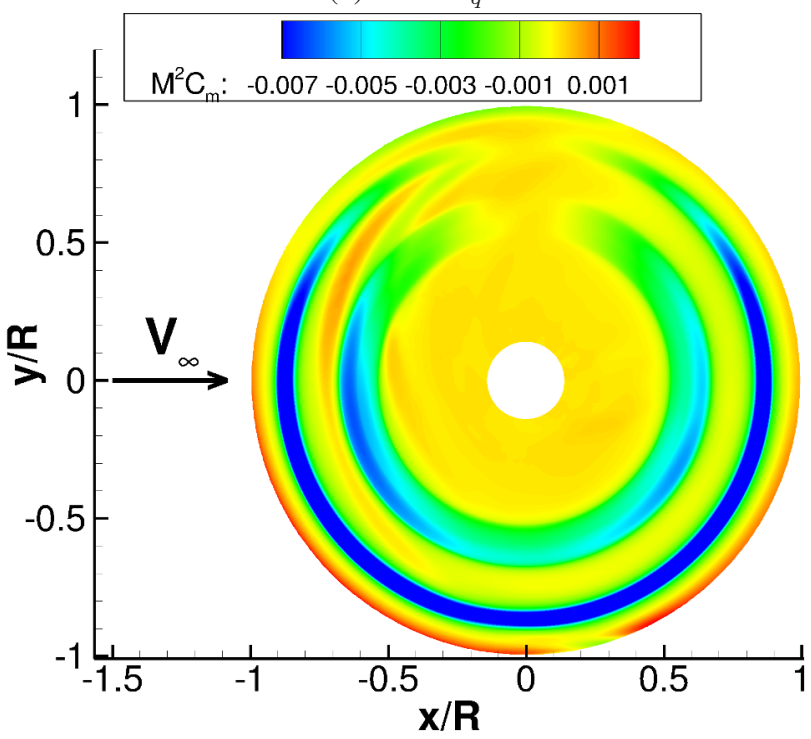

(f) $\Delta M^{2} C_{m}^{*}$

Figure 16: Mach-scaled normal force, torque and moment coefficient predictions for Flight 1-10. In the plots without flaps, thick black lines indicate a value of 0 . 


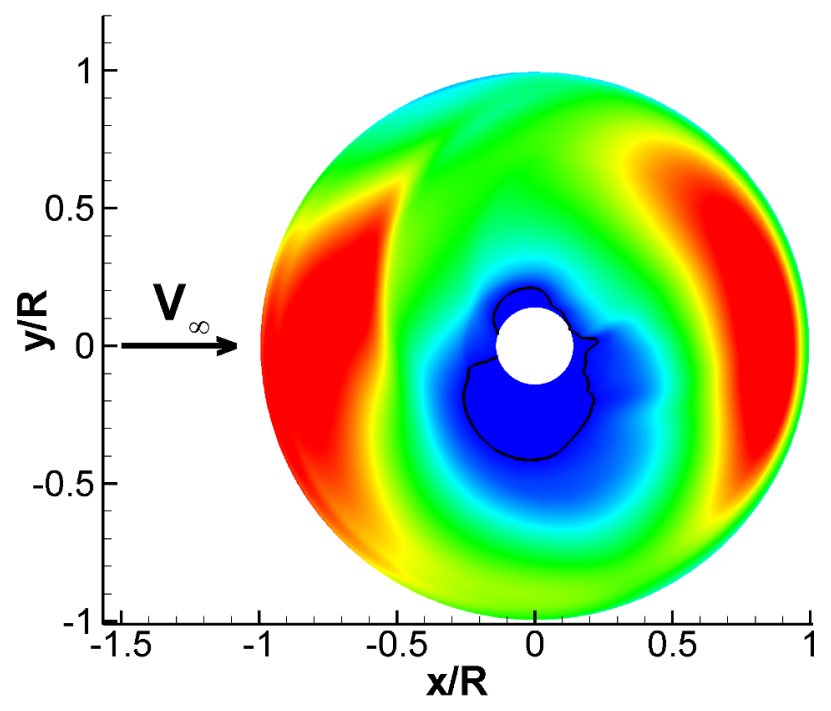

(a) $M^{2} C_{n}$, without flaps

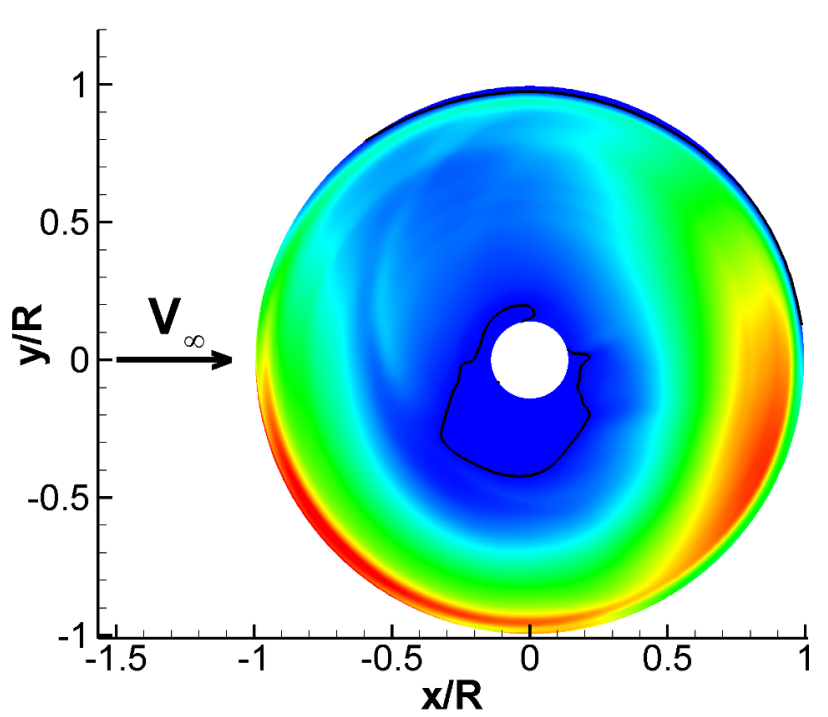

(c) $M^{2} C_{q}$, without flaps

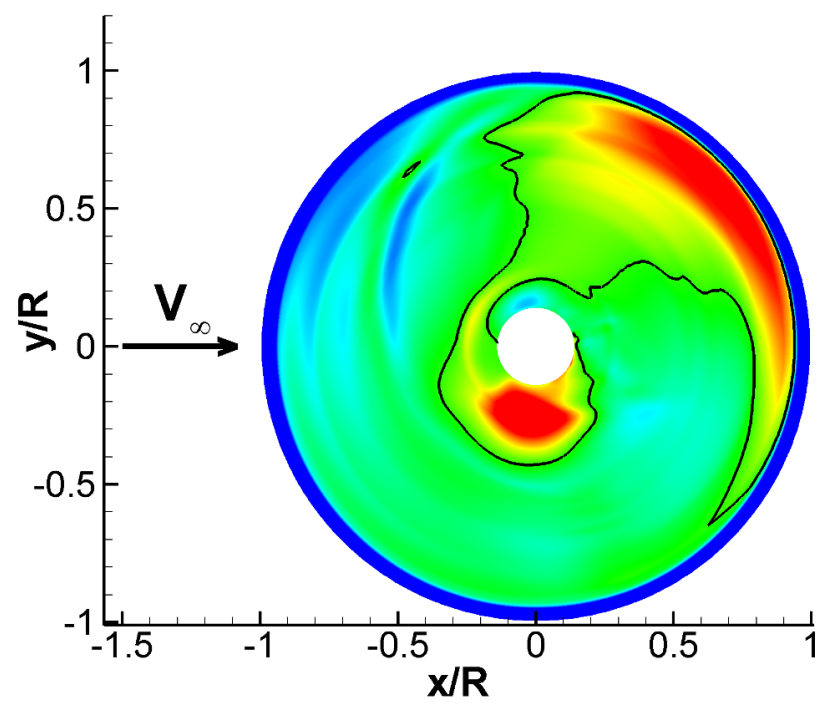

(e) $M^{2} C_{m}$, without flaps

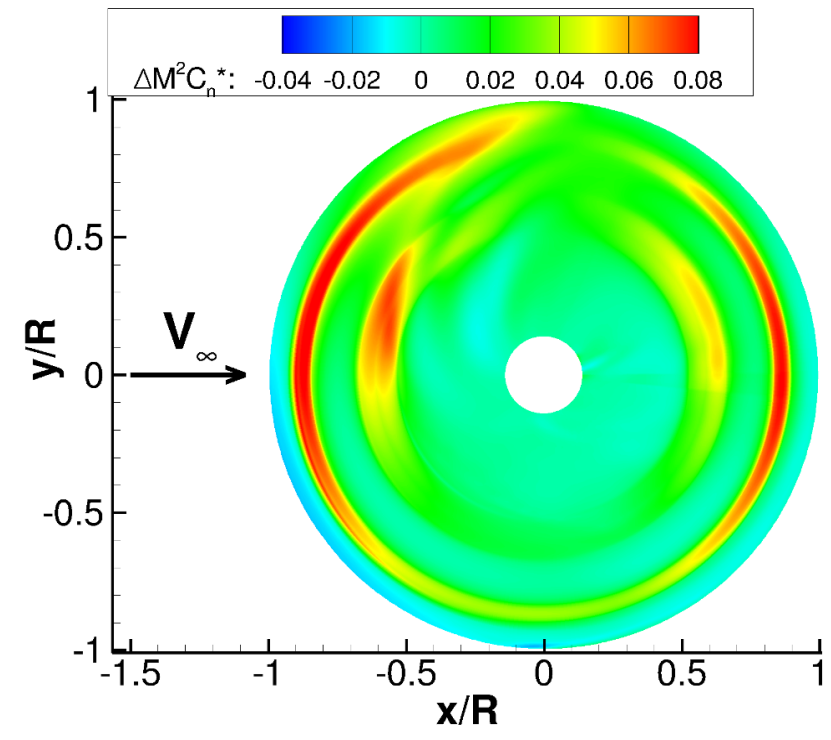

(b) $\Delta M^{2} C_{n}^{*}$

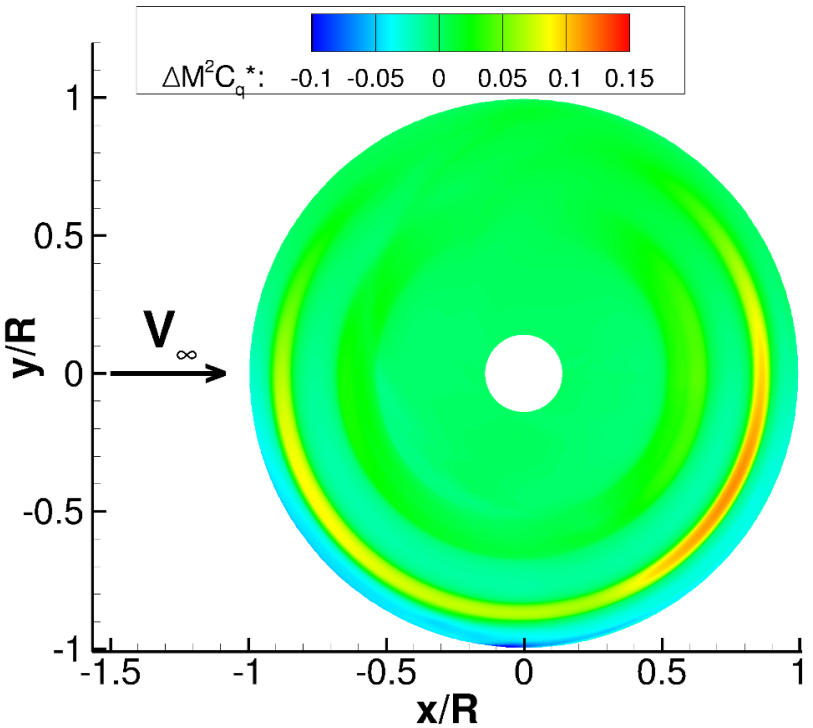

(d) $\Delta M^{2} C_{q}^{*}$

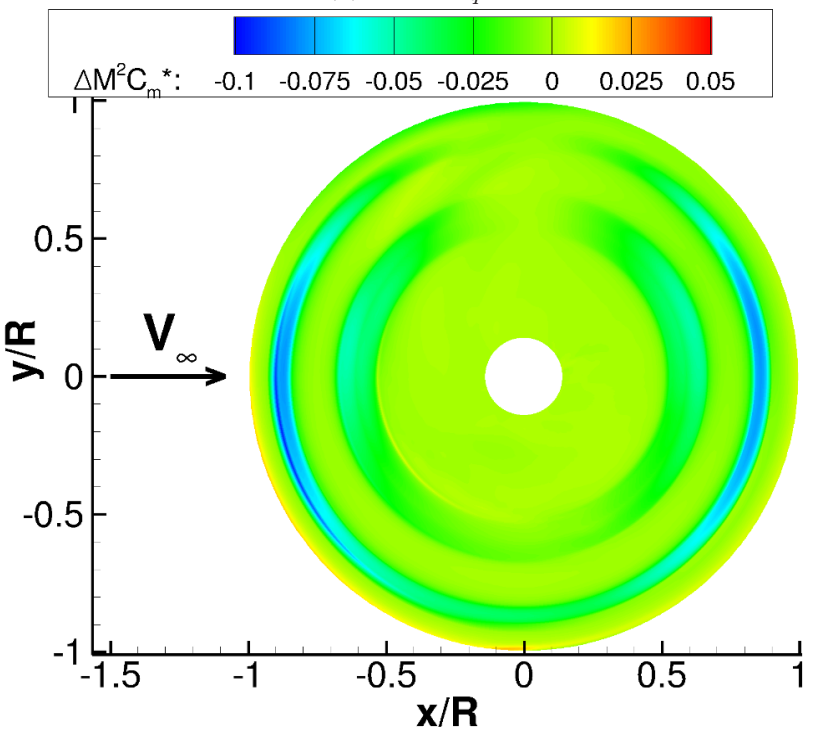

(f) $\Delta M^{2} C_{m}^{*}$

Figure 17: Mach-scaled normal force, torque and moment coefficient predictions for Flight 1-11. In the plots without flaps, thick black lines indicate a value of 0 . 


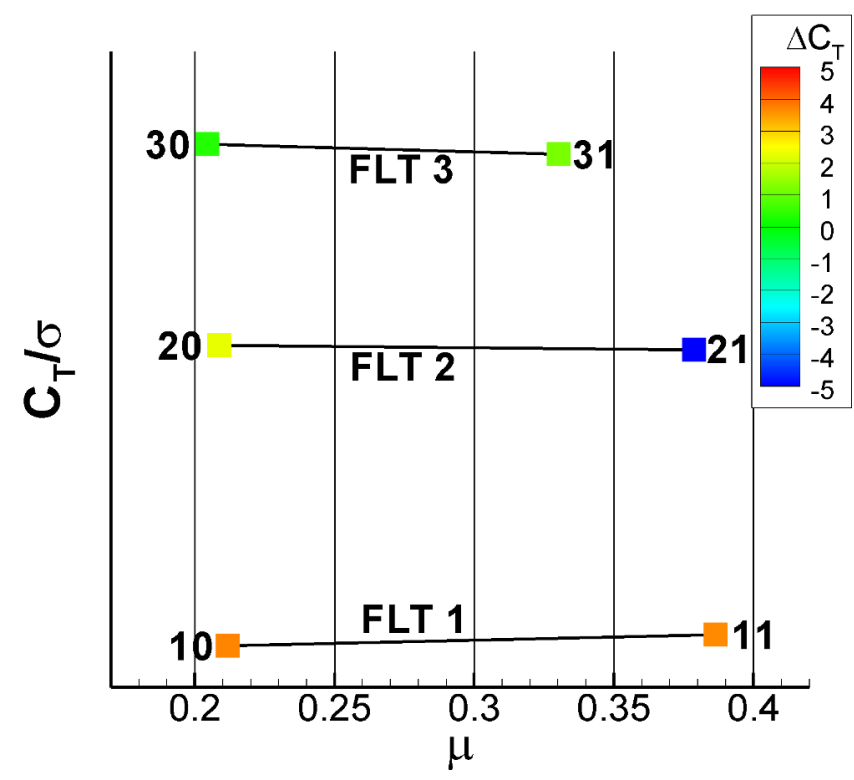

(a) $\Delta C_{T}$

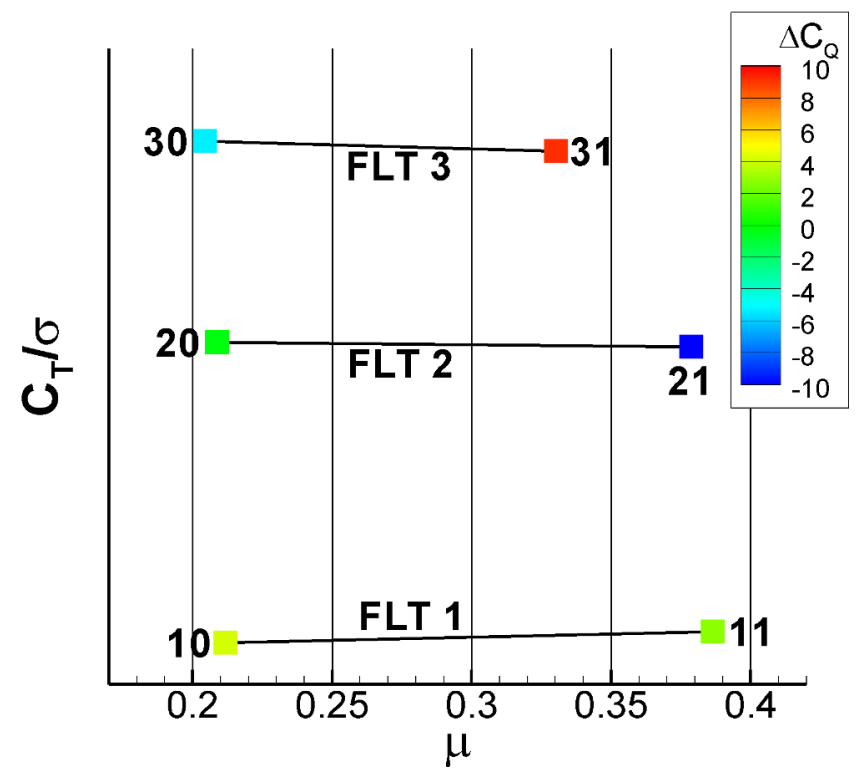

(b) $\Delta C_{Q}$

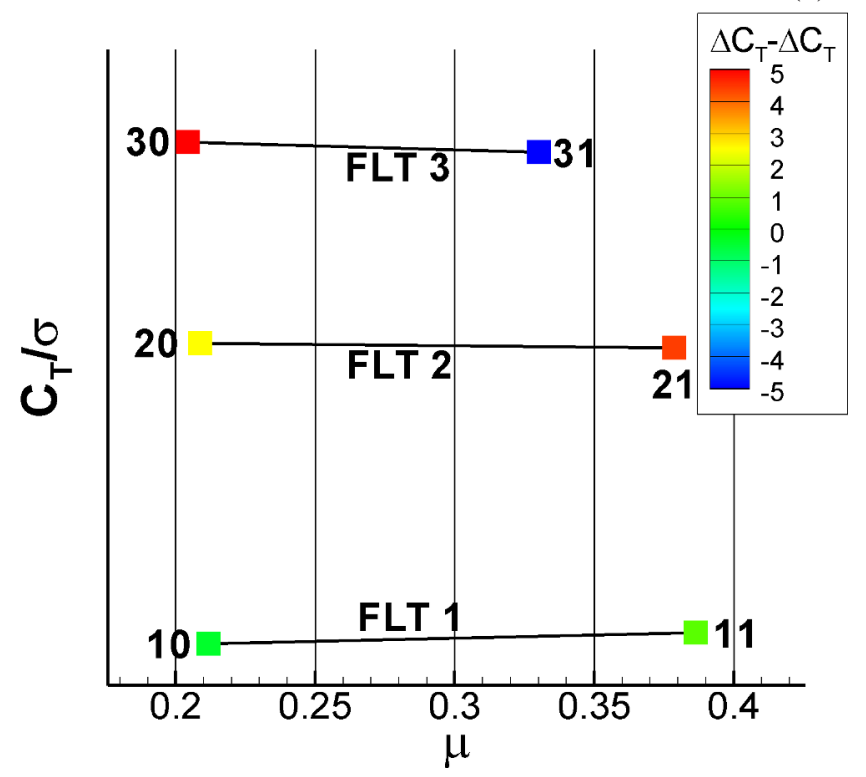

(c) $\Delta C_{T}-\Delta C_{Q}$

Figure 18: Visualisation of the evolution, in percents, of the thrust and torque coefficient in the flight domain with the flaps. 


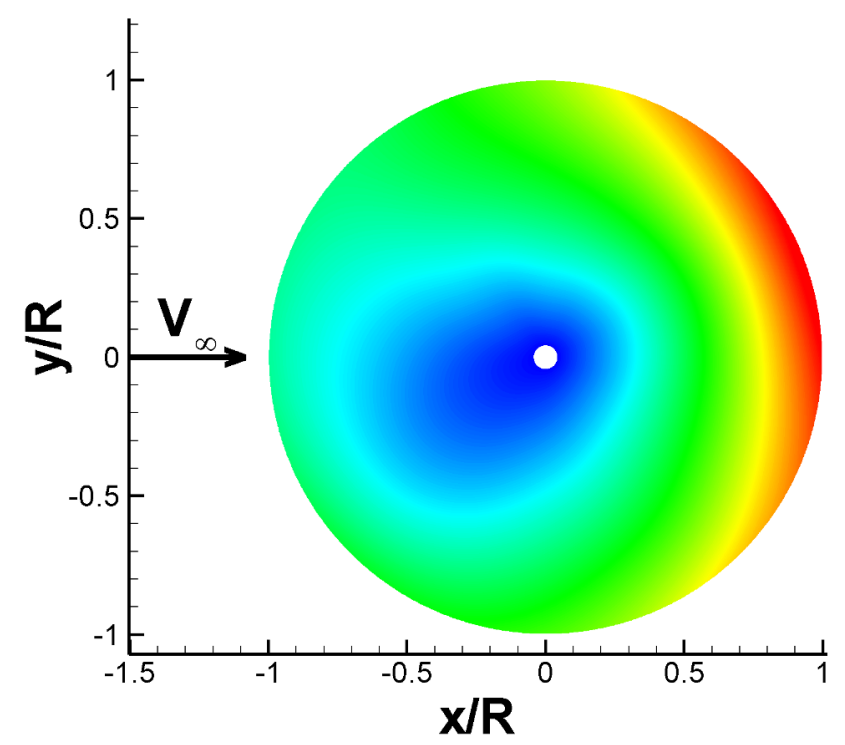

(a) $\Delta Z / R$

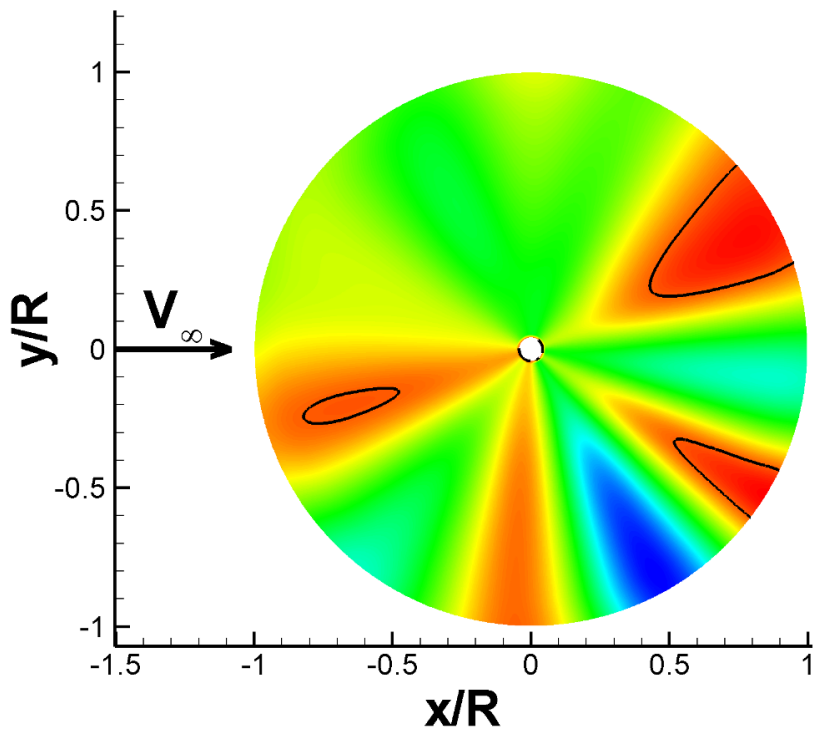

(b) $\theta_{\text {elas }}$

Figure 19: Prescribed deformation to the main rotor blade along the azimuth. 


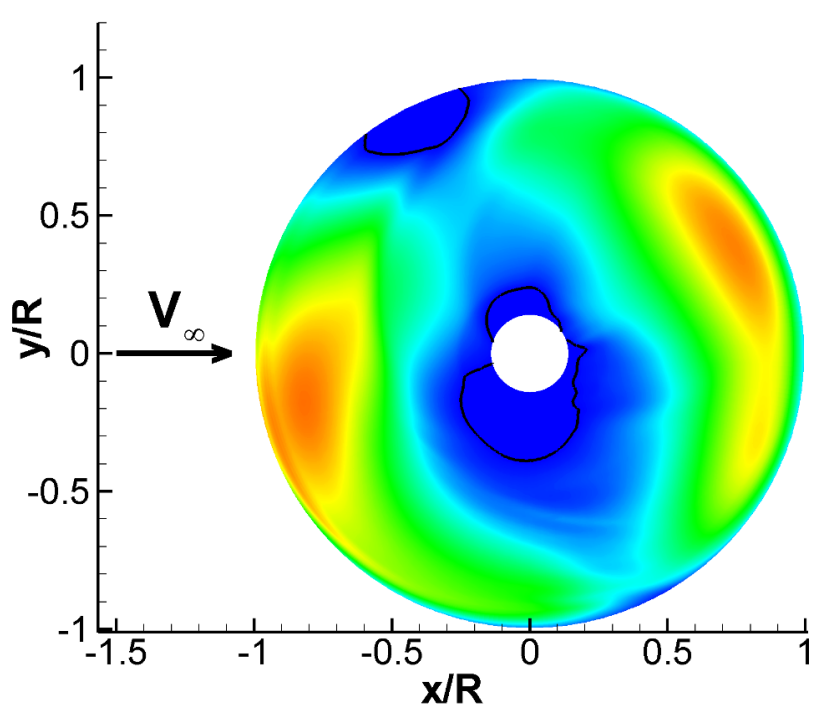

(a) $M^{2} C_{n}$, without flaps

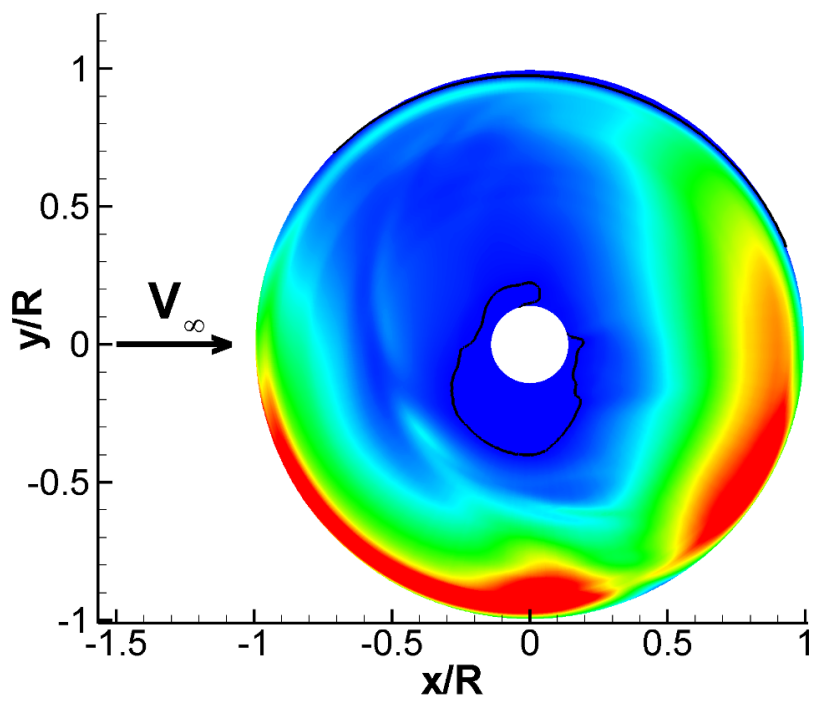

(c) $M^{2} C_{q}$, without flaps

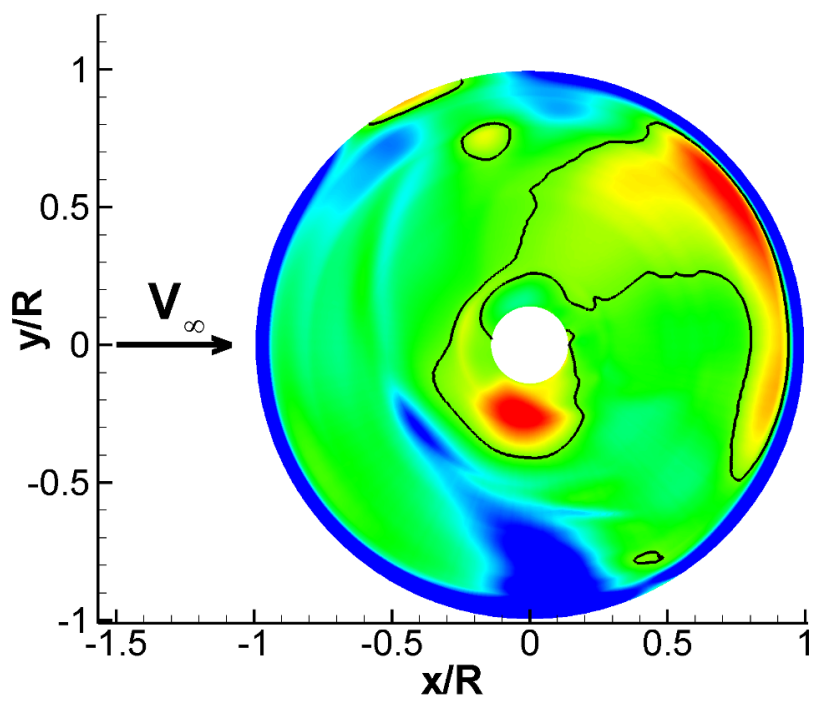

(e) $M^{2} C_{m}$, without flaps

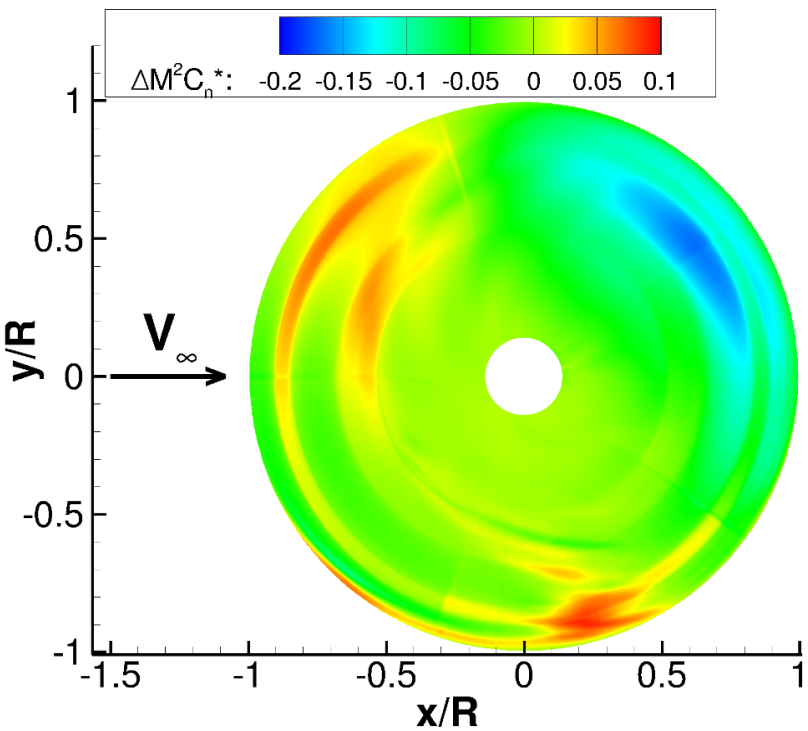

(b) $\Delta M^{2} C_{n}^{*}$

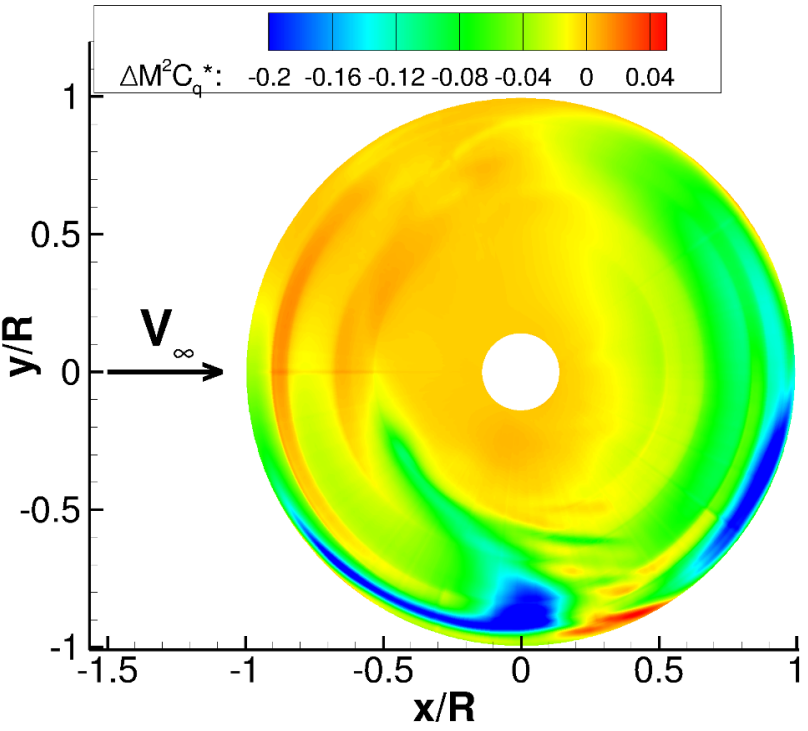

(d) $\Delta M^{2} C_{q}^{*}$

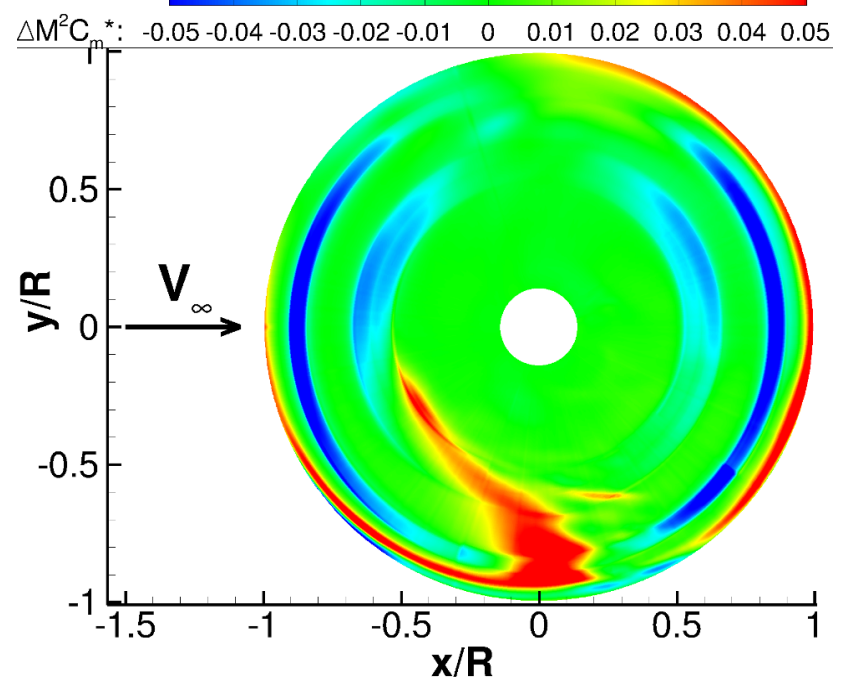

(f) $\Delta M^{2} C_{m}^{*}$

Figure 20: Mach-scaled normal force, torque and moment coefficient predictions for Flight 2-21, using prescribed deformations. Assessment of the effect of the flaps. Prescribed deformations were applied to the blades. In the plots without flaps, thick black lines indicate a value of 0 . 